\title{
ARP2/3- and resection-coupled genome reorganization facilitates translocations
}

Jennifer Zagelbaum ${ }^{1,2}$, Allana Schooley ${ }^{3 \wedge}$, Junfei Zhao ${ }^{4 \wedge}$, Benjamin R. Schrank ${ }^{1 \#}$, Elsa Callen ${ }^{5}$, Shan Zha ${ }^{1,6}$, Max E. Gottesman ${ }^{7}$, André Nussenzweig ${ }^{5}$, Raul Rabadan, Job Dekker ${ }^{3}$, and Jean Gautier ${ }^{1,8^{*}}$

${ }^{1}$ Institute for Cancer Genetics, Columbia University Vagelos College of Physicians and Surgeons, New York, New York, USA

${ }^{2}$ Integrated Program in Cellular, Molecular, and Biomedical Studies, Columbia University Vagelos College of Physicians and Surgeons, New York, New York, USA

${ }^{3}$ Department of Biochemistry and Biomedical Pharmacology, University of Massachusetts Medical School, Worcester, Massachusetts, USA

${ }^{4}$ Department of Systems Biology, Columbia University Vagelos College of Physicians and Surgeons, New York, New York, USA

${ }^{5}$ Laboratory of Genome Integrity, National Institutes of Health, Bethesda, Maryland, USA

${ }^{6}$ Department of Pathology and Cell Biology and Department of Pediatrics, Columbia University Vagelos College of Physicians and Surgeons, New York, New York, USA

${ }^{7}$ Department of Biochemistry and Biophysics, Columbia University Vagelos College of Physicians and Surgeons, New York, New York, USA

${ }^{8}$ Department of Genetics and Development, Columbia University Vagelos College of Physicians and Surgeons, New York, New York, USA

\#Current address: The University of Texas MD Anderson Cancer Center, Houston, Texas 77030

^ These authors contributed equally to this work

*Corresponding author

E-mail: jg130@cumc.columbia.edu 


\section{Summary}

2 DNA end-resection and nuclear actin-based movements orchestrate clustering of double-strand 3 breaks (DSBs) into homology-directed repair (HDR) domains. Here, we analyze how actin nucleation by

4 ARP2/3 affects damage-dependent and -independent 3D genome reorganization and facilitates 5 pathologic repair. We observe that DNA damage, followed by ARP2/3-dependent establishment of repair 6 domains enhances local chromatin insulation at a set of damage-proximal boundaries and affects 7 compartment organization genome-wide. Nuclear actin polymerization also promotes interactions 8 between DSBs, which in turn facilitates aberrant intra- and inter-chromosomal rearrangements. Notably, 9 BRCA1 deficiency, which decreases end-resection, DSB mobility, and subsequent HDR, nearly abrogates 10 recurrent translocations between AsiSI DSBs. In contrast, loss of functional BRCA1 yields unique 11 translocations genome-wide, reflecting a critical role in preventing spontaneous genome instability and 12 subsequent rearrangements. Our work establishes that the assembly of DSB repair domains is coordinated 13 with multiscale alterations in genome architecture that enable HDR despite increased risk of 14 translocations with pathologic potential.

\section{Main}

Eukaryotic nuclei are organized into functional domains enriched in proteins and factors involved

18 in nuclear transactions including RNA splicing, transcription, DNA replication, and repair ${ }^{1}$. Repair domains

19 assemble in biomolecular condensates mediated by mechanical forces and multivalent interactions

20 between proteins and nucleic acids ${ }^{1}$. We have shown that the actin nucleator, ARP2/3, and its activator

21 WASP promote clustering of DSBs into homology-directed repair (HDR) domains, which stimulates repair

22 by facilitating DNA end-resection ${ }^{2}$, the initial step of $\mathrm{HDR}^{3}$. In turn, resection leads to increased DSB

23 mobility ${ }^{2}$. Thus, HDR domains arise from the coordinated action of actin forces and repair reactions.

24 Besides facilitating repair $^{2,4-8}$, the impact of ARP2/3 and DSB-induced motion on genome 25 organization is poorly understood. Moreover, the pathologic consequences of assembling DSBs into 26 nuclear domains are not fully known. Imaging of DSBs in mammalian cells has revealed that translocating 27 breaks are mobile ${ }^{9}$, and clustering of DSBs in yeast promotes rearrangements ${ }^{6,10}$. Furthermore, CtIP28 dependent resection facilitates translocations in mouse cells ${ }^{11}$, highlighting that DSB end-processing can 29 lead to misrepair. Nevertheless, it remains unclear how proteins that mediate HDR domain formation, 30 including actin nucleators and resection machinery such as the tumor suppressor BRCA1, affect 31 chromosome translocations. 


\section{DNA damage induces local and global chromatin reorganization}

DNA damage activates the local, stepwise recruitment of repair proteins to damage sites as well as protein modifications that can spread over megabases along chromatinized DNA, such as the phosphorylation of the histone $\mathrm{H} 2 \mathrm{~A}$ variant, $\mathrm{H} 2 \mathrm{AX}^{12-14}$. Live-cell imaging and clustering analyses of DNA repair foci demonstrate that these processes translate into the $3 \mathrm{D}$ reorganization of chromatin ${ }^{2,15}$ but do not fully characterize the genomic features of repair domains and the rules that govern their assembly. To assess the impact of DSBs on genome organization, we performed chromosome conformation capture $(\mathrm{Hi}-\mathrm{C})$ in mouse embryonic fibroblasts $(\mathrm{MEFs})^{16}$ and human osteosarcoma cells (U2OS) ${ }^{17}$ harboring an inducible AsiSI restriction endonuclease. These cells express AsiSI fused to a truncated estrogen receptor that translocates to the nucleus upon induction with 4-hydroxy-tamoxifen (4OHT). There are over one thousand AsiSI recognition motifs in the mouse genome. However, cleavage efficiency, as measured by END-seq spike-in assays, revealed that a significant proportion of these sites are not cut in MEFs (Extended

Data Fig. 1a). Therefore, for quantitative analysis of $\mathrm{Hi}-\mathrm{C}$ data, we focused on a subset of 97 frequently cut AsiSI sites which showed the highest END-seq signal above background and collectively account for the approximately 100 DSBs per cell ${ }^{16}$. Cells were treated with the ARP2/3 inhibitor, CK-666, for six hours following induction of DSBs (+4OHT). After ensuring that CK-666 did not affect cutting efficiency of AsiSIER or the accumulation of damage (Extended Data Fig. 1b,c), two biological replicates were performed. Replicates of Hi-C experiments showed comparable phenotypes (Extended Data Fig. 1d), and data was pooled for the main figure sets.

$\mathrm{Hi}-\mathrm{C}$ studies have revealed that the genome can be split into A (open) and B (closed) chromatin

22 We first sought to examine the impact of DSBs and nuclear actin polymerization on A/B compartment 23 distribution using eigenvalue (principal component, PC1) decomposition of contact matrices ${ }^{18,19}$. 24 Strikingly, eigenvector analysis of intra-chromosomal interactions revealed that following induction of 25 damage, a significant fraction of $250 \mathrm{~kb}$ bins, particularly those with eigenvalues closer to zero, had a 26 relative increase in EV1 and apparent flip from B (closed chromatin) to A (open chromatin) (Fig. 1a;

27 Extended Data Fig. 2a,b). Approximately 15\% (all sites) or 25\% (top 97 sites) of B chromatin bins flipped 28 to the A compartment genome-wide or within $2 \mathrm{Mb}$ of frequently digested AsiS1 sites, respectively (Fig. 29 1a). These changes resulted in genome-wide enrichment of open chromatin with a nearly 10 percent 30 increase in the A compartment following damage (Extended Data Fig. 2a). An example of a B-to-A 31 compartment flip (blue to red) coinciding with an AsiSI site on chromosome 2 is shown (Fig. 1b, DSB \#50).

32 Switches were also found at a distance from the AsiSI site, as seen 2 Mb downstream of DSB \#8 (Fig. 1b). 
1 Notably, ARP2/3 inhibition with CK-666, dampened damage-induced compartment flips, indicating a role

2 for nuclear actin polymerization in genome compartmentalization (Fig. 1a; Extended Data Fig. 2a,b).

3 Compartment flips during transcriptional regulation correlate with changes in histone modifications ${ }^{20}$.

4 Similarly, restriction endonuclease damage induces megabase-sized chromatin remodeling events,

5 including $\mathrm{H} 2 \mathrm{AX}$ phosphorylation, ubiquitin accumulation, and histone $\mathrm{H} 1$ depletion ${ }^{14}$, which may

6 contribute to the compartment switching events seen here. We next visualized genome-wide

7 compartmentalization using saddle plots, which display interaction frequencies between pairs of $250 \mathrm{~kb}$

8 loci arranged according to their first eigen values (Extended Data Fig. 2c). Compartment strength was

9 calculated using $(A A+B B) /(A B+B A)$ to assess the preference for homotypic $(A-A, B-B)$ over heterotypic $(A-$

10 B) interactions ${ }^{21}$. Saddle plots and strength quantification revealed that damage increased both

11 homotypic interactions and compartment strength in the B compartment (Extended Data Fig. 2c).

12 Notably, ARP2/3 inhibition with CK-666 slightly increased B-B interaction strength in both the presence

13 and absence of damage, further suggesting a role for actin nucleation in compartmentalization (Extended

14 Data Fig. 2c).

15 Contact probability $P$ plotted as a function of genomic distance $s(P(s))$ can reveal properties of 16 chromatin architecture including the size and density of cohesin-dependent loops ${ }^{22}$ (Extended Data Fig.

$172 \mathrm{~d}$ ). The derivative of $P(s)$ typically displays a local peak at $s \sim 100 \mathrm{~kb}$, corresponding to the average size of

18 loops, followed by a valley at $s \sim 2 \mathrm{Mb}$. The depth of the valley is related to loop density 22 . Analysis of the

19 derivative of $P(s)$ for $\mathrm{Hi}-\mathrm{C}$ data obtained from cells with induced DNA damage revealed a more pronounced

20 valley at $s \sim 2 \mathrm{Mb}$ (Extended Data Fig. 2d). This can be interpreted as a general increase in loop density.

21 An increased number of loops/kb of DNA upon damage is consistent with previous observations of

22 increased cohesin recruitment to DSBs, including those induced by restriction endonucleases, that could

23 reflect cohesin-driven loop extrusion at $\mathrm{DSBs}^{23-25}$, or possibly more generally genome-wide. Indeed,

24 average $\mathrm{Hi}-\mathrm{C}$ interaction frequency aggregated at CTCF sites showed increased line formation (Extended

25 Data Fig. 2e). Such lines reflect increased cohesin-mediated loop formation where one base of the loop is

26 anchored at the CTCF sites. Hi-C interaction frequency between pairs of convergent CTCF-CTCF sites also

27 increased upon DNA damage (Extended Data Fig. 2f). Interestingly, the difference in $P(s)$ upon DNA

28 damage, as well as average insulation and loop strength at and between CTCF sites was not diminished in

29 the presence of CK-666 (data not shown).

30 Next, we aggregated contact matrices spanning $2 \mathrm{Mb}$ around frequently cut AsiSI sites to visualize

31 interactions in the absence (No Damage, N.D.) and presence $(+4 \mathrm{OHT}$ ) of DSBs (Fig. 1c). Average contact

32 maps revealed a striking level of organization in undamaged cells, as seen by strong insulation at the AsiSI 
motifs (Fig. 1c, left panel). Importantly, gene set-enrichment analysis (GSEA), which identifies statistically significant relationships between biological states, showed that frequently cut AsiSI sites are enriched in transcriptionally active areas (Extended Data Fig. $\mathbf{2 g}$ ). Upon induction of DSBs, average insulation at these sites increased in both MEFs (Fig. 1c, middle and right panels) and in AsiSI-U2OS cells (Extended Data Fig.

$\mathbf{2 h}$ ) indicating that this is a conserved feature. This increase is likely driven by a subset of DSBs located at insulated boundaries, or compartment boundaries. This suggests that DNA damage strengthens preexisting genome organization into more robust DNA repair domains ${ }^{15,25,26}$.

These data reveal multiscale changes in the 3D genomic landscape following damage, including compartment switching that favors open chromatin states, increased loop density, and reinforcement of pre-existing chromatin organization surrounding DSBs. It also suggests that long-range genome

11 reorganization, such as damage-induced compartment flipping that enriches for A compartment, is 12 regulated in part by ARP2/3-driven nuclear actin polymerization.

\section{Chromosome translocations occur at sites of DSB clustering}

In yeast and mammalian cells, DSB mobility drives clustering of DSBs into repair factories ${ }^{2,4-8}$.

16 Therefore, we sought to characterize long-range intrachromosomal interactions using aggregate peak 17 analysis (APA) ${ }^{27,28}$. We piled-up all possible pairwise interactions (304) occurring within chromosomes (in $18 \mathrm{cis}$ ) between the most frequently cut AsiSI sites. Following damage, we visualized distant DSBs coming 19 together in both MEF and U2OS cells (Fig. 2a, Extended Data Fig. 3a-d). DSB cluster enrichment scores 20 were calculated by comparing the average signal intensity at the center of the plot with that of the 21 surrounding area, allowing for relative quantification of interaction frequency between AsiSI sites.

22 Clustering increased upon addition of $4 \mathrm{OHT}$ (1.24 to 2.01), indicating that DNA damage triggered 23 increased interactions between distant DSBs within individual chromosomes (Fig 2a; Extended Data Fig. 24 3c). These distant DSB-DSB interactions were partially reduced by CK-666 treatment (2.01 to 1.80). These 25 data suggest that this DNA damage-dependent clustering is driven in part by ARP2/3-dependent nuclear 26 actin polymerization.

27 Next, we sought to visualize clustering of individual DSBs within a single chromosome (Chr 2). 28 We analyzed $\mathrm{Hi}-\mathrm{C}$ interactions (normalized to observed/expected) between the area surrounding a 29 frequently cleaved DSB on chromosome 2 and $1 \mathrm{Mb}$ bins spanning the rest of chromosome 2 . For 30 differential interaction plots, blue bars above the axis indicate strengthened interactions following 31 damage, while red bars below the axis represent a decrease in interaction frequency (Fig. 2b). 32 Concordant with APA analysis (Fig. 2a), we found that following damage this reference site (gray) 
1 interacted with other DSBs in cis (arrows) with frequencies significantly higher than in the absence of

2 damage (Fig. 2b, top). Next, we assessed the impact of DSB clustering in cis (Fig. 2b) on intrachromosomal

3 translocations. Indeed, a significant fraction of oncogenic translocations takes place between loci on the

4 same chromosome ${ }^{29-31}$. Thus, we performed high-throughput genome-wide translocation sequencing

5 (HTGTS) to test whether genomic reorganization following damage influences chromosome

6 rearrangements. HTGTS identifies translocation events between a fixed "bait" DSB and "prey" sites

7 throughout the genome (Extended Data Fig. 4a) ${ }^{32}$. To explore how damage-induced chromatin

8 reorganization influences aberrant rearrangements, we used the same AsiSI reference site on

9 chromosome 2 as the bait site. HTGTS analyses revealed that the loci of heightened interactions

10 corresponded to sites of frequent translocations with the bait (Fig. 2b, bottom).

11 We then assessed the correlation between contact frequency $(+4 \mathrm{OHT})$ and translocation

12 frequency across chromosome 2 . We observed that chromatin contact frequency predicted translocation

13 occurrence with a significant Pearson correlation coefficient, $r$, of 0.6490 (Fig. 2c). Thus, DNA repair

14 domains are sites where translocations can occur.

ARP2/3-mediated DSB clustering facilitates genomic rearrangements

Given that approximately 100 AsiSI sites are efficiently cut upon induction of AsiSI-ER, we

18 predicted that most recurrent translocations would take place between active AsiSI loci. Indeed, more

19 than $80 \%$ of prey originated from within 500 bp of an AsiSI site (Fig. 3a), whereas approximately $15 \%$ of

20 translocations occurred $10 \mathrm{~kb}-100 \mathrm{Mb}$ away from an AsiSI motif. The distribution of prey sites in U2OS

21 cells revealed similar classes (proximal, distal) of translocations (Extended Data Fig. 4b). Translocations

22 between AsiSI-proximal sites and the bait are recurrent, with variable levels of end-resection, mostly

23 under $500 \mathrm{bp}$. Recurrent translocations are not identical at the nucleotide level as PCR duplicates are

24 filtered by the HTGTS pipeline. Translocations to distal prey are primarily unique translocations

25 (Extended Data Fig. 4c) and might involve spontaneous, physiological DSBs forming at sites of intrinsic

26 genome fragility, including R-loops, G4 quadruplex, stalled replication forks, and active transcription ${ }^{33}$.

27 We showed that frequently cleaved AsiSI sites are within transcriptionally active regions (Extended Data

28 Fig. 2g). Similarly, translocations originating from loci proximal to AsiSI sites were highly enriched in

29 promoter sequences (Extended Data Fig. 4d) and located in transcriptionally active areas, as seen by

30 GSEA (Extended Data Fig. 4e). In contrast, translocations originating from regions distal to AsiSI sites

31 occurred throughout the genome (Extended Data Fig. 4d). 
ARP2/3 facilitates distant DSB-DSB interactions (Fig. 2a) and promotes clustering of repair foci².

2 However, it is not known whether nuclear actin dynamics impact the frequency of chromosome 3 translocations. To establish formally that increased interactions between DSBs drives chromosome rearrangements, we assessed the impact of ARP2/3 inhibition on translocations genome-wide, using HTGTS. Translocations were monitored six hours after DSB induction in control cells and in cells treated with CK-666. CK-666 significantly decreased the normalized frequency (see methods) of both intrachromosomal (chromosome 2), $p<0.01$ for the three binned loci (Fig. 3b) and inter-chromosomal translocations, $p=3 \cdot 6 \cdot 10^{-16}$ (Fig. 3c). Furthermore, the fold decrease (CK-666/DMSO) in normalized, AsiSIproximal translocation frequency was comparable for intra- and inter-chromosomal translocations (Extended Data Fig. 4f). This establishes that ARP2/3-dependent actin nucleation is a driving force for 11 chromosome rearrangements.

We then asked how DSB mobility affected translocations to spontaneous DSBs. The frequency of translocations to distal DSBs was significantly decreased following treatment with ARP2/3 inhibitor,

14 albeit to a lesser extent than the frequency of translocations to proximal DSBs (Fig. 3d). Thus, a smaller

15 fraction of physiologic translocations is driven by nuclear actin polymerization. The propensity to 16 translocate in the presence of CK-666 could reflect intrinsic properties of the prey loci, including their 17 transcriptional activity. Therefore, we examined the impact of ARP2/3 inhibition on translocations 18 originating from DSBs in promoter, gene body, and intergenic regions (Fig. 3e). ARP2/3 inhibition 19 significantly reduced the frequency of translocations arising from spontaneous DSBs in promoter regions, 20 a decrease that mirrored the effect of CK-666 on recurrent, experimentally-induced (AsiSI-AsiSI) 21 rearrangements (compare Fig. 3d and Fig. 3e). ARP2/3 inhibition also modestly decreased translocations

22 initiating from spontaneous DSBs in gene bodies. In contrast, ARP2/3 inhibition did not have a statistically 23 significant impact on translocations arising from intergenic loci.

DNA sequences at translocation junctions provide further insight into the repair mechanisms driving rearrangements ${ }^{34,35}$. Specifically, the presence of limited microhomology $(\mathrm{MH})$ suggests repair by alternative end-joining (alt-EJ) whereas blunt-end ligation indicates repair by classical NHEJ (c-NHEJ) ${ }^{36}$.

27 We found that only $18 \%$ of junctions resulted from blunt-end ligation (Extended Data Fig. 5a). In contrast, $2869 \%$ of junctions harbored microhomologies $(\mathrm{MH})$, emphasizing the importance of alt-EJ in mediating 29 pathologic repair (Extended Data Fig. 5a). Unexpectedly, we observed that $13 \%$ of translocations 30 contained additional short insertions. These complex rearrangements did not arise from direct ligation 31 of blunt ends or from annealing of staggered DNA ends between the bait and prey chromosomes 32 (Extended Data Fig. 5a). Of note, larger inserts (> $30 \mathrm{bp}$ ) were not detected due to the limits of HTGTS 
analysis, suggesting that insertions are more frequent than we report. To explore the origins of insertion events, we mapped inserts (20 bp $-30 \mathrm{bp})$ for all proximal reads. The majority of inserts $(>80 \%)$ mapped to the vicinity of prey loci, often on the antiparallel strand (Extended Data Fig. $\mathbf{5 b , c}$ ). This suggests that an intermediate step, possibly a transient invasion or annealing event, took place prior to the ligation that gave rise to a stable translocation.

\section{Distinct roles for BRCA1 in regulating translocations}

ARP2/3 clusters spontaneous and endonuclease-generated DSBs into HDR domains, where translocations occur. Inhibition of Mre11-dependent resection, the initial step of HDR, impairs DSB mobility and ARP2/3-mediated clustering ${ }^{2,15,37}$, highlighting a role for resection in the formation of repair

11 domains. In addition to promoting end-resection at HDR breaks, BRCA1 safe-guards against chromosome 12 translocations, as evidenced by the accumulation of genomic rearrangements in BRCA1-deficient 13 tumors ${ }^{38-40}$. Therefore, we sought to examine the impact of BRCA1 loss on chromosome mobility and 14 translocations. We used BRCA ${ }^{\Delta 11}$ AsiSI MEF cells with a truncated BRCA1 that lacks exon 11, impairing 15 DSB resection ${ }^{16,41}$. We first confirmed that cleavage at the chromosome 2 bait site was comparable in WT 16 and BRCA1 ${ }^{\Delta 11}$ cells (Extended Data Fig. 6a), then performed HTGTS in BRCA1-deficient MEFs. The 17 frequency of recurrent translocations, both intra-chromosomal (chromosome 2), p < 0.001 (Fig. 4a) and 18 inter-chromosomal, $\mathrm{p}=9.58 * 10^{-24}$ (Fig. 4b) between AsiSI-AsiSI DSBs was markedly decreased in $19 B R C A 1{ }^{\triangle 11} M E F s$. This finding is consistent with the role of BRCA1 in promoting end-resection, which occurs 20 upstream of ARP2/3 activity. Indeed, inhibition of ARP2/3 in BRCA1-deficient cells did not further reduce 21 translocation frequency (Fig. 4a). We next evaluated the link between DSB resection and mobility by

22 performing live-cell imaging of BRCA1 ${ }^{\Delta 11}$ MEFs. We found that mean square displacement (MSD) of NBS1

23 repair foci, which is recruited prior to resection of DSBs, was substantially lower in BRCA1-deficient cells

24 as compared to WT (Fig. 4c). These findings further strengthen the idea that recurrent translocations are 25 facilitated within HDR domains, the site of BRCA1 action.

Whole genome sequencing of tumors harboring BRCA1 mutations has revealed rearrangement

27 signatures thought to be the consequence of BRCA1's role in suppressing endogenous genome 28 instability ${ }^{38,40}$. Therefore, we next examined how BRCA1-deficiency might specifically affect spontaneous 29 translocations by analyzing the distribution of prey as a function of distance to the nearest AsiSI site (Fig. 30 4d). Strikingly, translocations in BRCA1 ${ }^{\Delta 11}$ cells occurred more frequently between the bait and distal 31 DSBs than in WT cells +/- CK-666 (Fig. 4d,e; Extended Data Fig. 6b-d), indicating a distinct role of BRCA1 32 in preventing translocations. Furthermore, analysis of the cumulative frequency of prey distribution in 
1 WT and BRCA $1^{\Delta 11}$ cells as a function of the distance to AsiSI sites revealed significantly different

2 distributions (Extended Data Fig. 6b). This increase in translocations to distant loci could manifest from

3 replication fork collapse or transcription-related stress as both processes are resolved by intact BRCA1 $1^{42,43}$

4 (Extended Data Fig. 6e).

\section{Conclusions}

DNA damage triggers local signaling to facilitate repair reactions at DNA lesions ${ }^{44}$, subsequent checkpoint activation yielding long-range histone and post-translational modifications ${ }^{45}$, and ARP2/3mediated DSB mobility ${ }^{2,4}$. Together, these events promote the formation of HDR domains. Our studies provide insights into the coordinated, multiscale reorganization of the 3D genome leading to the

11 formation of these domains. First, we observe local strengthening of insulation boundaries at DSBs ${ }^{25,26}$

12 (Fig. 1c; Extended Data Fig. 7, 1) and increased chromatin loop extrusion at CTCF boundaries ${ }^{46-48}$

13 (Extended Data Fig. 2d-f), both possibly the result of increased cohesin loading. Second, we provide a

14 genomic view of DSB clustering (Fig. 2a; Extended Data Fig. 7, 2). Finally, we document DNA damage-

15 dependent, genome-wide changes in compartmentalization that can be quantified as B to A compartment

16 flips (Fig 1a, b, Extended Data Fig. 7, 3). Notably, damage-induced, long-range reorganization, such as

17 clustering of DSBs and compartment flips, is facilitated in part by ARP2/3-dependent forces, whereas local

18 changes in insulation and loop extrusion are not. These data are consistent with a model in which

19 chromatin accessibility following damage is favored within A compartments. In turn, this facilitates DSB

20 clustering and the generation of HDR domains, while repair activity surrounding individual DSBs is 21 restricted by enhanced insulation.

22 Using high throughput translocation assays (HTGTS), we show that ARP2/3- and resection-

23 mediated formation of HDR domains increases the risk of chromosomal translocations while facilitating

24 homologous recombination ${ }^{2}$. The increased contact frequency revealed by $\mathrm{Hi}-\mathrm{C}$ is not just due to 25 rearrangements as translocation events are much more rare. We confirm that HTGTS is a powerful 26 method for identifying translocations to naturally unstable loci, establishing that ARP2/3's impact on 27 chromosomal rearrangements is not limited to restriction endonuclease-generated DSBs but is also 28 relevant for physiological damage. Nevertheless, the partial impact of ARP $2 / 3$ inhibition points to 29 additional mechanisms for DSB clustering and pathogenic translocations, which may include different 30 actin nucleators ${ }^{15,49}$, alternate cytoskeleton proteins $s^{4,50}$, and phase-separated boundaries ${ }^{51,52}$.

31 Chromosome translocations require an end-joining step ${ }^{53}$ (data not shown). Here we establish 32 that clustering of resected DNA ends arising from transcriptionally active loci is also critical for 
1 translocations. We thus propose that translocations are generated by a two-step process. First, actin

2 nucleators (ARP2/3) and resection machinery (BRCA1) bring recurrent and spontaneous DSBs harboring

3 resected ends into close proximity (Extended Data Fig. 7, 2). Second, resected DNA ends are processed to

4 be compatible with end-joining reactions or alternatively, transiently invade the prey locus capturing

5 additional sequences prior to end-joining. This is consistent with frequent insertion events observed

6 previously at translocation junctions $s^{54,55}$ as well as in this study (Extended Data Fig. 5b). Finally, we

7 establish that while BRCA1-dependent resection facilitates DSB mobility, increasing translocations

8 between recurrent DSBs, the tumor suppressor maintains genome integrity during DNA transactions,

9 preventing spontaneous translocations to fragile genomic regions (Extended Data Fig. 7). Overall, our

10 work highlights the delicate balance between faithful repair and misrepair at play within HDR domains

11 and the critical roles of actin nucleators and repair proteins in achieving this balance.

\section{Methods}

14 Cell culture and drug treatment

15 Mouse embryonic fibroblast (MEF) and U2OS cell lines were cultured in high-glucose Dulbecco's modified 16 Eagle's medium supplemented with L-glutamine, 10\% fetal bovine serum, and 1\% penicillin-streptomycin. 17 ER-AsiSI MEF cell lines, including WT and BRCA1 ${ }^{\Delta 11}$ cells, were developed as previously described ${ }^{16}$. Cells 18 were treated with doxycycline (Sigma-Aldrich: D3072, $3 \mu \mathrm{g} / \mathrm{mL}$ ) for 24 hours to induce AsiSl expression.

19 4-OHT (Sigma-Aldrich: H7904, $1 \mu \mathrm{g} / \mathrm{mL}$ ) was added for the last 6 hours of doxycycline treatment to induce 20 AsiSI translocation. Cells were co-treated with DMSO or $100 \mu$ M CK-666 (Sigma Aldrich: SML-006, $100 \mu M$ ) 21 and incubated at $37^{\circ} \mathrm{C}$ for 6 hours.

22 ER-AsisI U2OS cells were obtained from Dr. Gaelle Legube ${ }^{13}$. For cell synchronization, cells were 23 treated with $2 \mathrm{mM}$ thymidine for two 18-h intervals separated by an 11-h release in fresh medium. 24 Following double-thymidine block, cells were released into fresh medium for $7 \mathrm{~h}$ (G2) or $15 \mathrm{~h}$ (G1). 25 Exponentially growing or synchronized cells were treated with $300 \mathrm{nM} \mathrm{4-OHT} \mathrm{(Sigma} \mathrm{Aldrich,} \mathrm{H7904)} \mathrm{to}$ 26 induce damage with DMSO or $100 \mu \mathrm{M} \mathrm{CK}-666$. Cells were incubated at $37^{\circ} \mathrm{C}$ for 24 hours.

\section{$\mathrm{Hi}-\mathrm{C}$}

29 Chromosome conformation capture experiments were performed as previously described ${ }^{33}$ with some 30 modifications. Briefly, 5 million cells/library were crosslinked with $1 \%$ formaldehyde and lysed. After 31 digesting chromatin with 400 units of Dpnll overnight, DNA ends were labeled with biotinylated dATP 32 using 50 units Klenow DNA polymerase. Blunt-end ligation was performed with 50 units T4 Ligase at $16^{\circ} \mathrm{C}$ 
1 for 4 hours, followed by reverse crosslinking with $400 \mu \mathrm{g} / \mathrm{ml}$ proteinase $\mathrm{k}$ at $65^{\circ} \mathrm{C}$ overnight. DNA was 2 purified using phenol/chloroform extraction and ethanol precipitation, and concentrated on a $30 \mathrm{kDa}$ 3 Amicon Ultra column. Biotin was removed from unligated ends in $50 \mu l$ reactions using 50 units T4 DNA polymerase/5 mg DNA. Following DNA sonication (Covaris S220) and Ampure XP size fractionation to generate DNA fragments of 100-300 bp, DNA ends were repaired using 7.5U T4 DNA polymerase, 25U T4 polynucleotide kinase, and 2.5 U Klenow DNA polymerase. Libraries were enriched for ligation products by biotin pulldown with MyOne streptavidin C1 beads. To prepare for sequencing, A-tailing was performed using 15 units of Klenow DNA polymerase (3'-5' exo-) and Illumina TruSeq DNA LT kit Set A indexed adapters were ligated. Libraries were amplified in PCR reactions for 5-7 cycles and subjected to

10 Ampure XP size selection prior to sequencing on an Illumina HiSeq 4000 machine using the Paired End 50 11 bp module. Two biological replicates were performed for each condition.

\section{Hi-C Analysis}

14 Paired-end 50bp reads were processed using the distiller pipeline ${ }^{56}$. First, reads from MEF and U2OS

15 libraries were mapped to $\mathrm{mm} 10$ and hg19 reference genomes, respectively, using BWA-MEM in single 16 sided mode (-SP). Alignments were then parsed, classified, and filtered using pairtools ${ }^{56}$. The resulting 17 valid pairs included uniquely mapped and rescued pairs with a minimum mapping quality of 30 . Valid pairs 18 were aggregated into binned contact matrices and kept as multi-resolution cooler files ${ }^{57}$ for subsequent 19 analyses. Where indicated, paired reads from replicate libraries were pooled prior to filtering for PCR 20 duplicates. All $\mathrm{Hi}-\mathrm{C}$ contact matrices were normalized by iterative correction ${ }^{19}$, excluding the first 2

21 diagonals. Downstream analyses were performed using cooltools version $0.3 .2^{58}$ unless otherwise 22 indicated, python 3.7.10, and matplotlib (Hunter, 2007). Hi-C interaction heatmaps were generated from 23 balanced $250 \mathrm{~kb}$ resolution coolerfiles using cooler "show". For heat maps, pooled and individual replicate 24 library sets were downsampled to equal read depth.

The average contact probability $(P(s))$ as a function of genomic distance was calculated using

26 "compute-expected" from cooltools version 0.4.059. The "diagsum" function was applied to balanced data

27 binned at $1 \mathrm{~kb}$ to compute expected, which was then parsed into log-spaced bins of genomic distance 28 using "logbin-expected". The rate of contact frequency decay as genomic distance increases, the $P(s)$ 29 derivative, was determined using "combine_binned_expected" and provides a highly informative 30 representation of $\mathrm{Hi}-\mathrm{C}$ data.

31 Active and inactive chromatin compartments were assessed based on eigenvector decomposition 32 of observed/expected cis contact matrices binned at $250 \mathrm{~kb}$ resolution using the cooltools "call- 
compartments" function. In this case, the first eigenvector, EV1, positively correlated with gene density and assignment of A or B compartment identity was based on high or low gene density, respectively. Saddle plots for cis interactions were generated using cooltools "compute-saddle". For each library, ranked EV1 values were binned into 30 quantiles and observed/expected interactions were plotted. Saddle strength was quantified by comparing the average interaction frequency of each AA or BB quantile bin or bins to the analogous $A B$ and $B A$ bins (effectually $(A B+B A) / 2$ ).

Average observed/expected $\mathrm{Hi}-\mathrm{C}$ interaction frequencies at subsets of genomic loci were determined using the cooltools "snipping" function. To examine DSB clustering, all pairwise cis interactions between bin-aligned Top97-digested AsiS1 sites were aggregated at $25 \mathrm{~kb}$ resolution with a $2 \mathrm{Mb}$ flanking window. The DSB cluster enrichment score was calculated by taking the ratio of the average $\mathrm{Hi}-\mathrm{C}$ interaction frequency in the $5 \times 5$ central bins ( $25 \mathrm{~kb}$ radius) and the average interaction frequency of the remaining bins (125 $\mathrm{kb}-1 \mathrm{Mb}$ radius). Loop extrusion was explored by examining aggregate $\mathrm{Hi}-\mathrm{C}$ interactions at CTCF sites. CTCF positions were determined using a previously published CTCF ChIP-seq dataset from MEFs ${ }^{60}$ (sample GSM2635593). Peaks were called using MACS3 (https://github.com/macs3project/MACS) with the default "callpeak" parameters and candidate CTCF motifs, generated in HOMER ${ }^{61}$ using a published vertebrate consensus ${ }^{62}$ within 200 bp of these peaks were selected. For pileups, top CTCF sites (13927 total) were flipped based on the direction of the consensus motif and aggregated at 5 $\mathrm{kb}$ bin resolution with a $100 \mathrm{~kb}$ flanking window. Loop aggregate plots were generated by considering all possible pairwise combinations of convergent CTCF sites on cis chromosomes with a genomic distance of 20-1000 kb (64044 possible loops). Loop scores were calculated by taking the ratio of the average Hi-C interaction frequency in the $5 \times 5$ central bins ( $25 \mathrm{~kb}$ radius) and the average interaction frequency of the remaining bins (25-100 $\mathrm{kb}$ radius).

\section{High-throughput Genome-wide Translocation Sequencing}

25 HTGTS was performed as previously described ${ }^{32}$. Briefly, genomic DNA was collected using 26 phenol/chloroform extraction, sonicated (Covaris S220), and amplified using biotin (MEF: 5' Bio27 TGgagAgCGATGAACTGGATC 3'; U2OS: 5'-Bio-GCCGACCAATAGCATGGCG- 3') and nested (MEF: 5'28 NNNNNNBarcodeCGAAAACAGGATCCCGCAGC-3'; U2OS: 5'-BarcodeACTGCGGCTGCATCCAATC-3') primers 29 targeting chromosome 2 (MEFs, chr2: 13271321) and chromosome 9 (U2OS, chr9: 130693175). For the 30 nested primer in MEF experiments, random nucleotides were added before the barcode to increase 31 library diversity. Sequencing was performed on an Illumina MiSeq sequencer. 


\section{$1 \quad$ High-throughput Genome-wide Translocation Analysis}

2 Burrows-Wheeler Aligner was used to align sequences to the mm10 (MEF) or hg19 (human) genomes.

3 Using established pipelines (https://github.com/robinmeyers/transloc pipeline), reads were filtered

4 with the default parameters. All reads had good mapping quality (mapping quality $>30$ ). For translocation

5 frequency, final reads were binned by $100 \mathrm{~kb}$ windows genome-wide. For each experiment, the number

6 of reads in each window was normalized to the corresponding number of bait-only sequences obtained

7 from the pipeline allowing us to compare translocation frequency between libraries. Genome

8 coordinates of prey sequences were annotated using R package ChIPseeker ${ }^{63}$, which retrieved the

9 location of each prey sequence (Promoter, Gene Body or Intergenic Region). Microhomology (MH)

10 analysis was performed as previously described ${ }^{35}$. $\mathrm{MH}$ was defined as the overlapping homologous

11 sequence between the bait and the prey site.

\section{Live-cell Imaging}

14 MEF cells were transfected with plasmids expressing NBS1-GFP using Neon Transfection System (1350 V,

$1530 \mathrm{~ms}, 1$ pulse). Cells were cultured on 35-mm glass bottom microwell dishes (MatTek) and damaged with

$160.5 \mu \mathrm{g} / \mathrm{ml} \mathrm{NCS}$ (Sigma N9162) for 60 minutes at $37^{\circ} \mathrm{C}$. Following two washes with PBS, cells were allowed

17 to recover for 10 hours before imaging. Imaging was performed on an A1RMP confocal microscope (Nikon

18 Instruments), on a TiE Eclipse stand equipped with a 60×/1.49 Apo-TIRF oil-immersion objective lens, an

19 automated XY stage, stage-mounted piezoelectric focus drive, and a heated, humidified stage top

20 chamber with 5\% CO2 atmosphere. Z series were collected at 0.4- $\mu \mathrm{m}$ intervals throughout the entire

21 nucleus every 5 min for 1 hour. Focus was maintained by the Perfect Focus System (Nikon). Mean-squared

22 displacement analysis was performed as previously described ${ }^{2}$.

\section{Immunohistochemistry and quantification of $\gamma \mathrm{H} 2 \mathrm{AX}$ foci}

25 Fixed-cell imaging experiments were performed as previously described ${ }^{2}$. Briefly, U2OS cells were cultured 26 on 8-well chamber slides and treated with $0.5 \mu \mathrm{g} / \mathrm{ml} \mathrm{NCS}$ for 60 minutes at $37^{\circ} \mathrm{C}$. Following two washes, 27 cells were incubated at $37^{\circ} \mathrm{C}$ to allow formation of $\gamma \mathrm{H} 2 \mathrm{AX}$ foci in the presence of DMSO or $100 \mu \mathrm{M}$ CK28 666. Cells were fixed with 4\% PFA ( $\mathrm{pH} 7.4)$ and permeabilized with $0.1 \%$ PBS-Trition X-100. Cells then were 29 treated with primary antibody ( $\mathrm{H} 2 \mathrm{AX}$, EMD Millipore: 05-636, 1/500) at $4{ }^{\circ} \mathrm{C}$ overnight and secondary 30 antibody (Alexa 488 conjugated goat anti-mouse Ig (Abcam: ab150113, 1/1,000)) for 1 hour at room 31 temperature. Cells were imaged under 40x magnification using a Zeiss Axio Imager Z2 microscope, 
1 equipped with a CoolCube1 camera (Carl Zeiss). Foci counting was performed using automated MetaCyte

2 software (Metasystems, version 3.10.6).

3

4 Quantification of AsiSI-induced DSBs

5 END-seq experiments and spike-in assays were performed as previously described ${ }^{16,64}$. AsiSI cutting

6 efficiency at specific sites was measured by quantitative polymerase chain reaction (qPCR $)^{17}$ using delta-

7 delta $\mathrm{Ct}$ to compare samples +/- 4OHT. Primers used in U2OS cells are as follows. Site 1: 5'-

8 GTCCCTCGAAGGGAGCAC-3', 5'-CCGACTTTGCTGTGTGACC-3'; Site 2: 5'-CCGCCAGAAAGTTTCCTAGA-3', 5'-

9 CTCACCCTTGCAGCACTTG-3'. Primers used in MEF cells are as follows. Bait: 5'-

10 TGGAGAGCGATGAACTGGATC-3', 5'-TGGCCGGATTTTGTGTGC-3'. Ct was normalized for DNA content using

11 primers distant from any AsiSI motifs (No DSB). In U2OS cells: 5'-ATTGGGTATCTGCGTCTAGTGAGG-3', 5'-

12 GACTCAATTACATCCCTGCAGCT-3'. In MEF cells: 5'-GGACAATGACCGCGTGTTTT-3', 5'-

13 AACAGCAGGCGCTCTATACC-3'.

15 Gene Set Enrichment Analysis

16 GSEAPreranked was used to assess the enrichment of the frequently cut AsiSI sites in transcriptionally 17 active regions. The transcriptional profile of MEF cell line was downloaded from GEO 18 https://www.ncbi.nlm.nih.gov/geo/query/acc.cgi?acc=GSE29278 ${ }^{65}$ to create the preranked gene list with

19 the level of gene expression as the input. The closest gene to each AsISI site was collected to make the

20 gene set ${ }^{*}$.gmt file. The same approach was used to assess enrichment of prey sites (HTGTS) in 21 transcriptionally active regions.

23 Data Availability: High throughput sequencing data have been deposited to Gene Expression Omnibus

24 under accession number GSEXXXXXX.

Acknowledgements: We thank the Molecular Cytogenetics and Molecular Pathology Shared Resources of

27 the Herbert Irving Comprehensive Cancer Center (HICCC) as well as T. Swayne and E.L. Munteanu from

28 the Confocal and Specialized Microscopy Shared Resource of the HICCC at Columbia University. We also

29 thank G. Legube for the ER-AsiSI U2OS cell lines; R. Baer for BRCA1 ${ }^{\triangle 11}$ MEFs used for live-cell imaging; and

30 J. Min, G. Sidhu, and Y. Rose for comments on the manuscript. This work was supported by the following

$31 \mathrm{NIH/NCI/NHGRI} \mathrm{grants:} \mathrm{F30-CA250166} \mathrm{(J.Z.),} \mathrm{CA197606} \mathrm{(J.G.),} \mathrm{CA174653} \mathrm{(J.G.,} \mathrm{R.R.,} \mathrm{and} \mathrm{Ju.Z),} \mathrm{and}$ 
1 HG003143 (J.D.). The A.N. laboratory is supported by the Intramural Research Program of the NIH. J.D. is

2 an investigator of the Howard Hughes Medical Institute.

3

4 Author Contributions: J.G. and J.Z. conceived of the study and wrote the manuscript. J.Z. conducted the

5 majority of experiments. J.Z. A.S., and Ju.Z. performed data analysis. J.D., R.R., S.Z., and M.G. aided with

6 data interpretation. A.S. and J.D. helped with implementation of $\mathrm{Hi}-\mathrm{C}$ protocols. B.S. aided with initial

7 experiments. E.C. and A.N performed END-seq experiments. 


\section{Figure Legends}
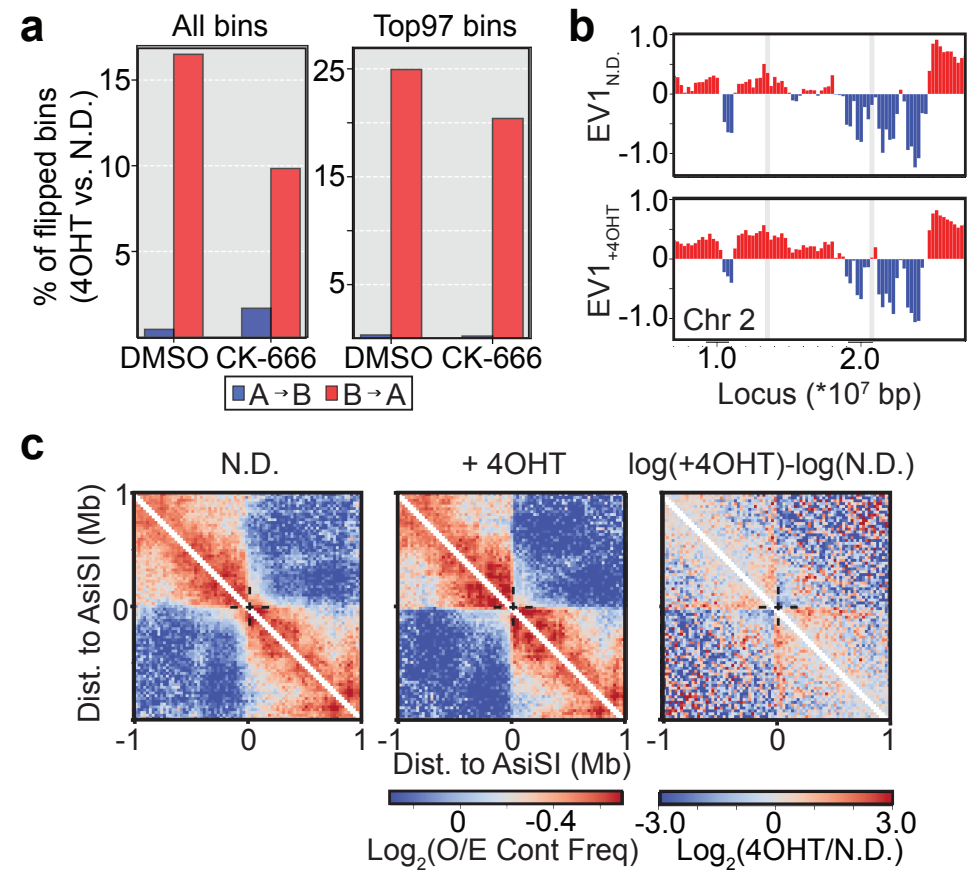

Figure 1| DNA damage induces multiscale alterations of the 3D genome. a, Percent of $A$ (open) or $B$ (closed) compartment bins ( $250 \mathrm{~kb}$ ) that flip identity genome-wide (left) upon induction of damage with 4OHT genome-wide and for the $2 \mathrm{Mb}$ regions surrounding the top 97 frequently cut AsiSI sites in MEFs (right). b, Representative trajectory of compartment flipping events. First eigenvector (EV1) tracks for cis interactions (250 kb bins) normalized to observed-expected. Values are phased by gene density (Active chromatin/A compartment>0, red). Frequently cut AsISI sites are highlighted in grey. c, Average $\log _{2}$ (observed/expected) $\mathrm{Hi}-\mathrm{C}$ interaction frequency maps of the aggregated $2 \mathrm{Mb}$ regions surrounding the most frequently cut AsiSI sites binned at $25 \mathrm{~kb}$ resolution. 

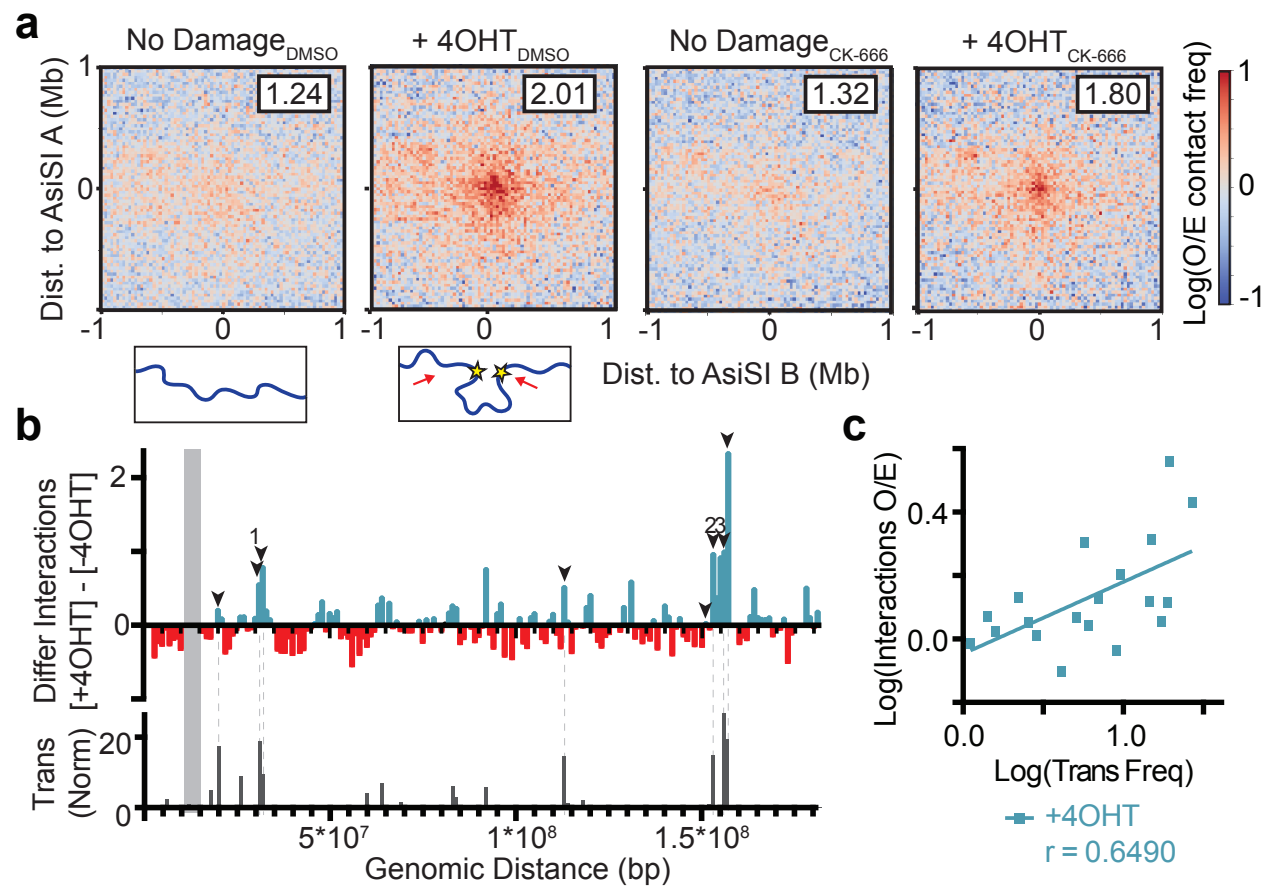

b

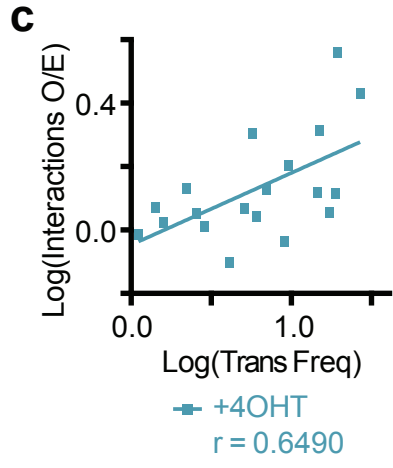

Figure 2| Translocations occur at sites of DSB clustering. a, Top: Aggregate peak analysis (APA) displaying the contact frequencies of all possible pairwise combinations of the the top 97 AsiSI digested sites in cis (304 interactions between damaged bins). Data is binned at $25 \mathrm{~kb}$ and averaged for a $2 \mathrm{Mb}$ flanking window. $\log _{2}$ (observed/expected) Hi-C maps are shown in the presence or absence of damage (4OHT) +/CK-666. For each APA plot, a cluster enrichment score is calculated using the ratios of the average interaction frequency of the 9 central bins $(125 \mathrm{~kb})$ / average interaction frequency of the outside bins (125 kb - $1 \mathrm{Mb}$ ). Bottom: Schematic visualization of intra-chromosomal interactions and clustering following damage. b, Top: Differential interaction plots normalized to observed/expected between a 1 $\mathrm{Mb}$ region surrounding the bait site on chromosome 2 and the rest of chromosome 2, +/- 4OHT. Arrows represent frequently cut AsiSI sites. Data adjacent to the bait site along the main diagonal (grey box) has been omitted (11000000 to $16000000 \mathrm{Mb}$ ). Numbers (1-3) indicate three intrachromosomal sites analyzed in Fig. 3b. Bottom: Normalized translocation frequency (translocations per 1,000 events in the dataset) between bait and chromosome 2 loci following damage. c, Log-log correlation plot of translocation frequency versus $\mathrm{Hi}-\mathrm{C}$ interaction frequency (+4OHT) for the 19 most common prey sites on chromosome 2. Pearson correlation (" $r$ ") is shown. Points within $1 \mathrm{Mb}$ of the bait site have been excluded. 

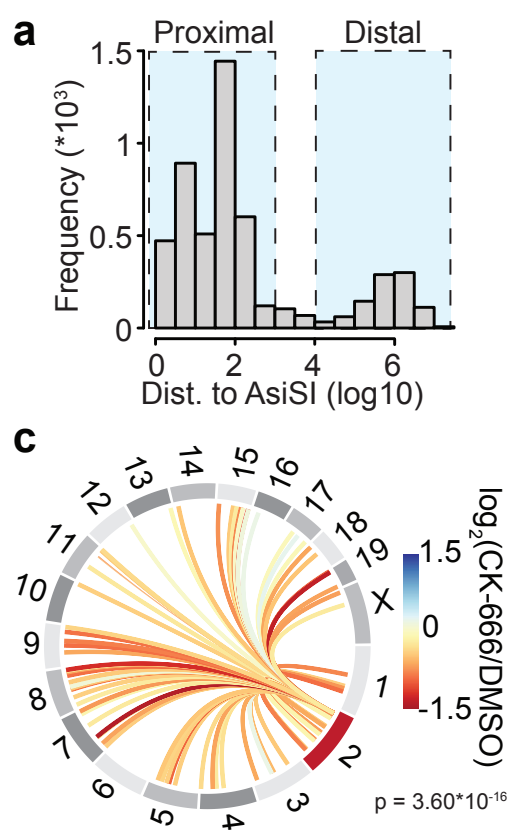

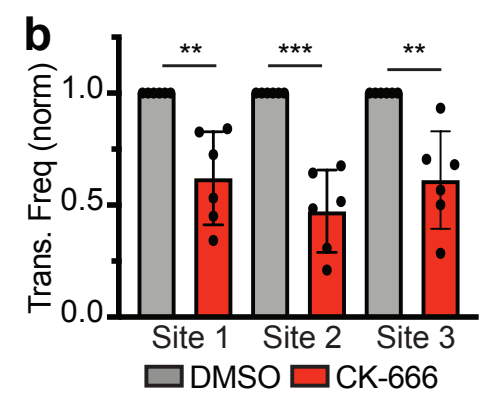

d



e

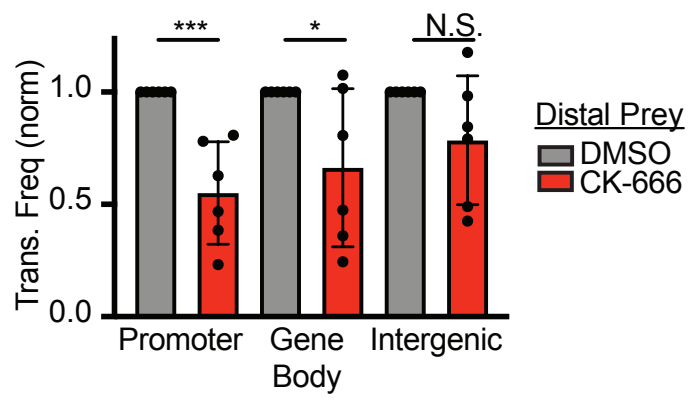

Figure 3 | ARP2/3-mediated clustering facilitates chromosomal translocations. a, Plot of all translocations as a function of their distance to the nearest AsiSI motif. Data is divided into proximal $(<500$ bp of an AsiSI site) and distal (>10 kb from an AsiSI site) prey b, Normalized translocation frequency at three sites on chromosome 2 ( $1=31,900,000-32,000,000$ bp; $2=153,600,000-153,700,000$ bp; $3=$ 156,100,000-156,200,000 bp) in WT MEF AsiSI cells +/- 100 MM CK-666. $P$ calculated by Student's twotailed t-test. Mean and standard deviation. c, Circos plot visualizing differential normalized translocation frequencies genome-wide following damage in the presence or absence of ARP2/3 inhibitor, CK-666 (100 $\mu \mathrm{M})$ at binned loci that had $\geq 10$ translocation events. Connecting lines are colored according to the $\log _{2}$ fold change following damage between +/- CK-666 populations. Chromosome 2 (red) contains the bait site. $P=3.60 * 10^{-16}$, Wilcoxon test. $\mathbf{d}$, Normalized translocation frequencies to proximal $(<500 \mathrm{bp}$ from AsiSI site) and distal (>10 kb from AsiSI site) loci in the presence and absence of $100 \mu \mathrm{M}$ CK-666. $P$ calculated by Student's two-tailed t-test. Mean and standard deviation. e, Normalized translocation frequencies for distal prey in promoter, gene body, and intergenic regions in the presence or absence of CK-666. $P$ calculated by Student's two-tailed $t$-test. Mean and standard deviation. 


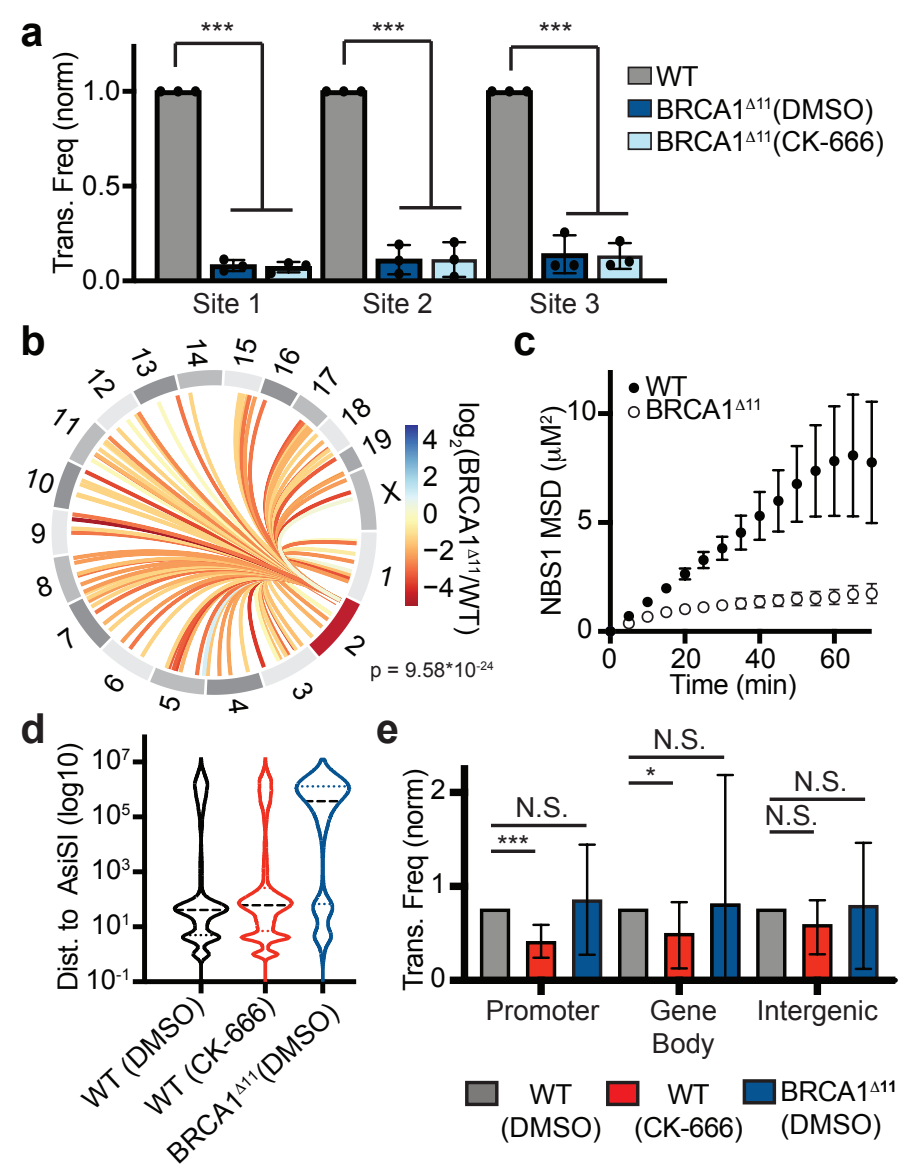

Figure 4| Distinct roles for BRCA1 in regulating translocations. a Figure 4| Distinct roles for BRCA1 in regulating translocations. a, Normalized translocation frequencies at three binned sites on chromosome $2(1=31,900,000-32,000,000$ bp; $2=153,600,000-153,700,000$ bp; $3=156,100,000-156,200,000$ bp $)$ in WT and BRCA1 ${ }^{\Delta 11}$ MEF AsiSI cells +/- $100 \mu \mathrm{M}$ CK-666. Columns are normalized to frequency of translocations in WT cells in the same biological replicate. $P$ calculated by one-way ANOVA Tukey's multiple comparisons. Mean and standard deviation. b, Circos plot showing differential normalized translocation frequencies following damage in BRCA ${ }^{\Delta 11}$ cells compared to WT. Connecting lines are colored according to the $\log _{2}$ fold change between WT and BRCA1-deficient cell types following damage. Chromosome 2 (red) contains the bait site. $P=9.58 * 10^{-24}$, Wilcoxon test. c, Mean-squared displacement of NBS1-GFP foci in WT and BRCA $1^{\Delta 11}$ cells treated with $0.5 \mu \mathrm{g} / \mathrm{ml}$ NCS. $n>195$ foci in $>12$ nuclei. d, Violin plot displaying the distribution of translocating prey as a function of the distance to the nearest AsiSI site in WT cells +/- CK-666 and BRCA1 ${ }^{\Delta 11}$ cells (dashed line $=$ median, dotted lines $=$ quartiles, $\mathrm{n}>4$ biological replicates). e, Normalized translocation frequencies for distal prey in promoter, gene body, and intergenic categories in WT cells (+/- CK-666) and BRCA1 ${ }^{\Delta 11}$ cells. $P$ calculated with Student's two-tailed t-test. Mean and standard deviation. 


\section{Extended Data Figure Legends}

a

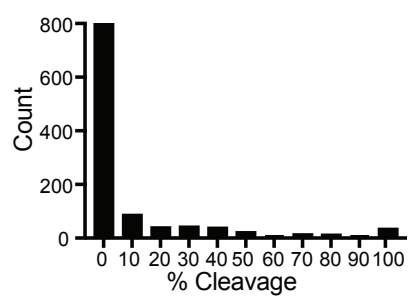

b

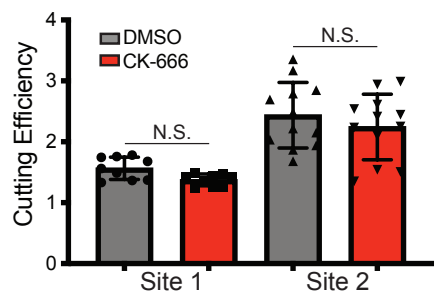

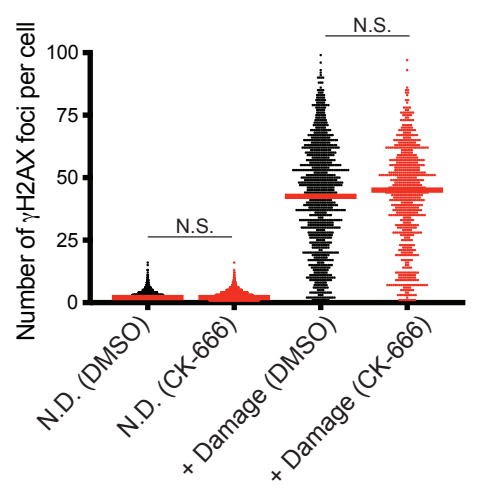

d $+4 \mathrm{OHT}-\mathrm{R} 1$ $+4 \mathrm{OHT}-\mathrm{R} 2$

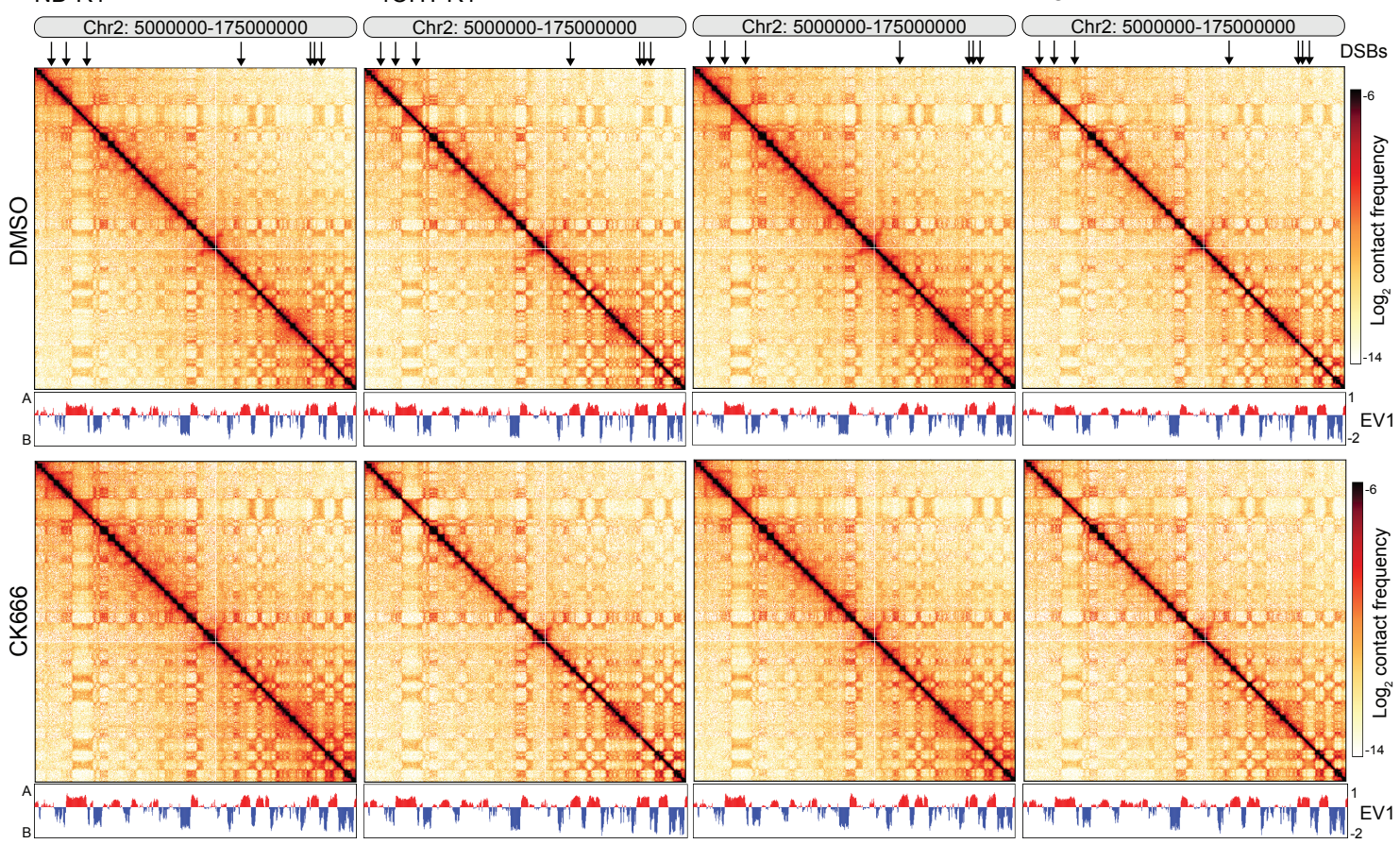

Extended Data Figure 1| Characterization of MEF and U2OS cell lines. a, AsiSI restriction endonuclease cutting efficiency at all AsiSI motifs in MEFs as measured by END-seq spike-in assays. $\mathbf{b}$, Cutting efficiency for two AsiSI sites (chr9: 130693175 and chr2: 38864106) in U2OS cells +/- $100 \mu$ M CK-666. DNA was extracted from cells 4 hours following damage and \% DSBs was measured using quantitative PCR amplification with primers close to the AsiSI sites, normalized to a control (uncleaved) site. Mean and standard deviation. $\mathrm{n}=3$ biological replicates with each 3 technical replicates. $P$ calculated by Student's two-tailed t-test. c, Quantification of $\gamma \mathrm{H} 2 \mathrm{AX}$ foci/cell in undamaged U2OS cells, and cells treated with 0.5 $\mu \mathrm{g} / \mathrm{ml} \mathrm{NCS}+/-\mathrm{CK}-666$. Cells were allowed to recover for two hours following damage. $P$ calculated by twosided Mann-Whitney test. Red line indicates mean. d, Hi-C interaction frequency maps for a region of chromosome 2 binned at $250 \mathrm{~kb}$ and accompanying first eigenvector traks (EV1) for cis interactions phased 
bioRxiv preprint doi: https://doi.org/10.1101/2021.10.22.465487; this version posted October 24, 2021. The copyright holder for this preprint (which was not certified by peer review) is the author/funder, who has granted bioRxiv a license to display the preprint in perpetuity. It is made available under aCC-BY-NC-ND 4.0 International license.

by gene density (Active/A compartment $>0$ ). Top 97 frequently digested AsiSI sites in MEFs are indicated by arrows. 
a

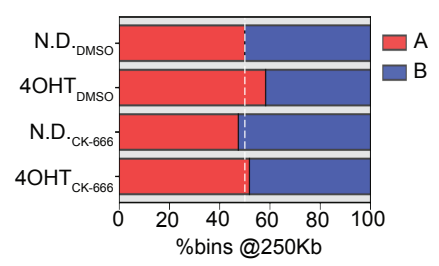

C

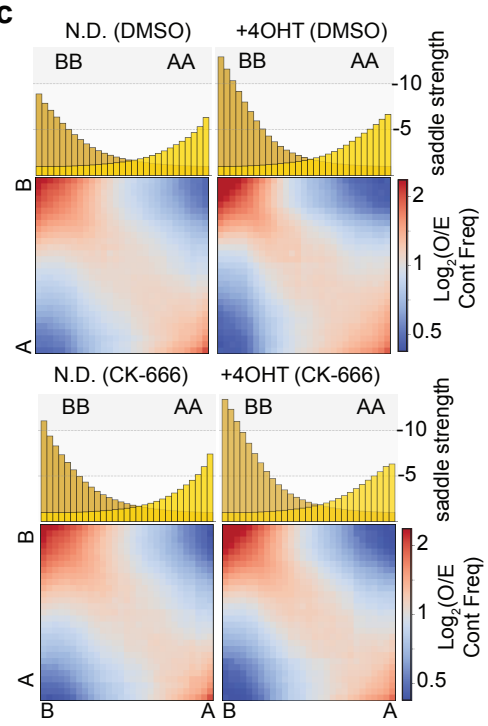

g

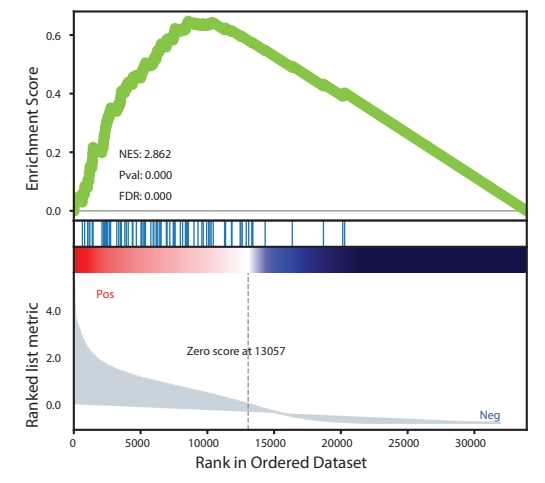

d b
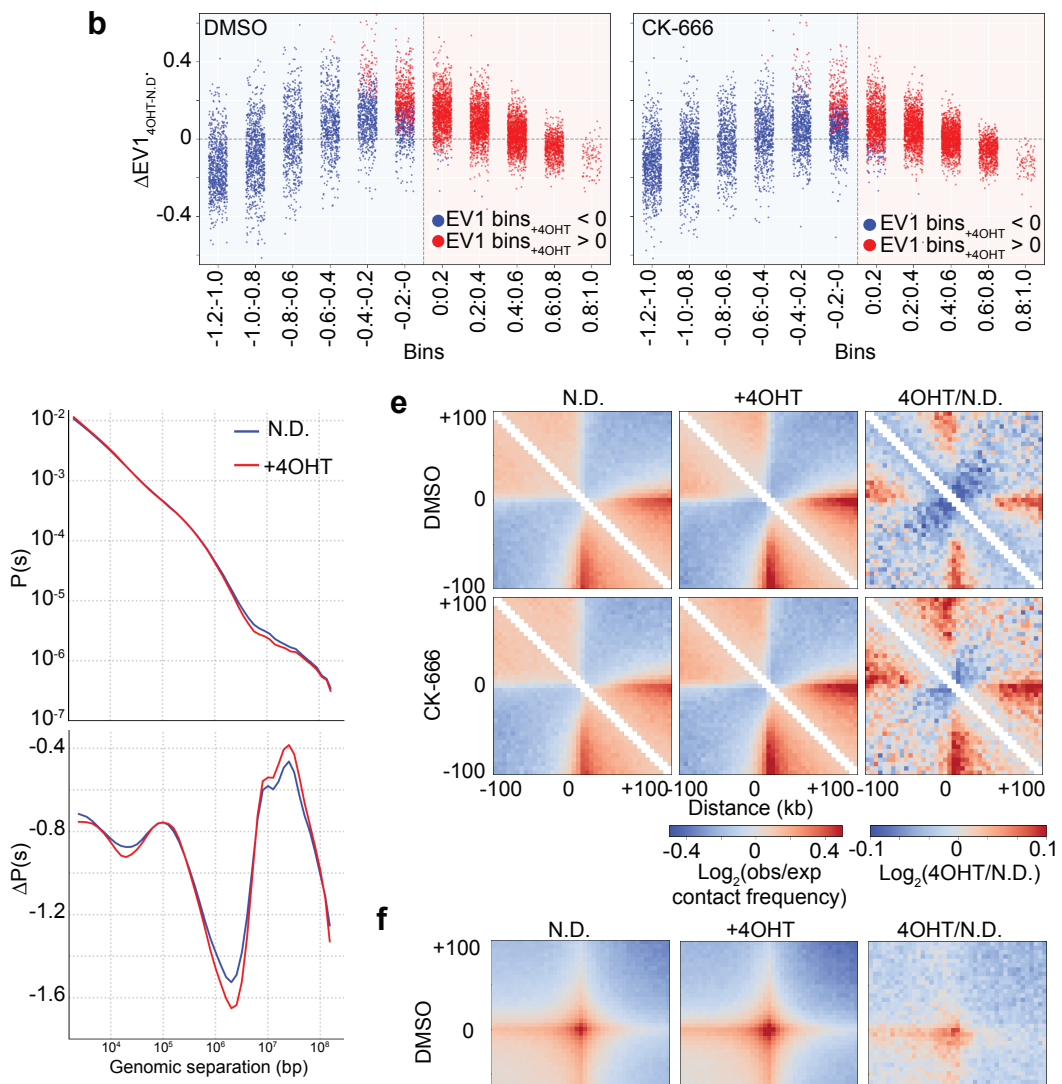

e N.D.

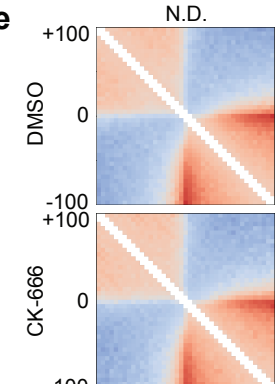

$+4 \mathrm{OHT}$

4OHT/N.D.

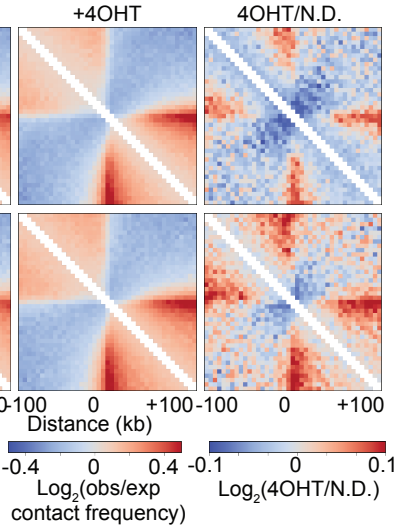

$f$
0
D.
0
0
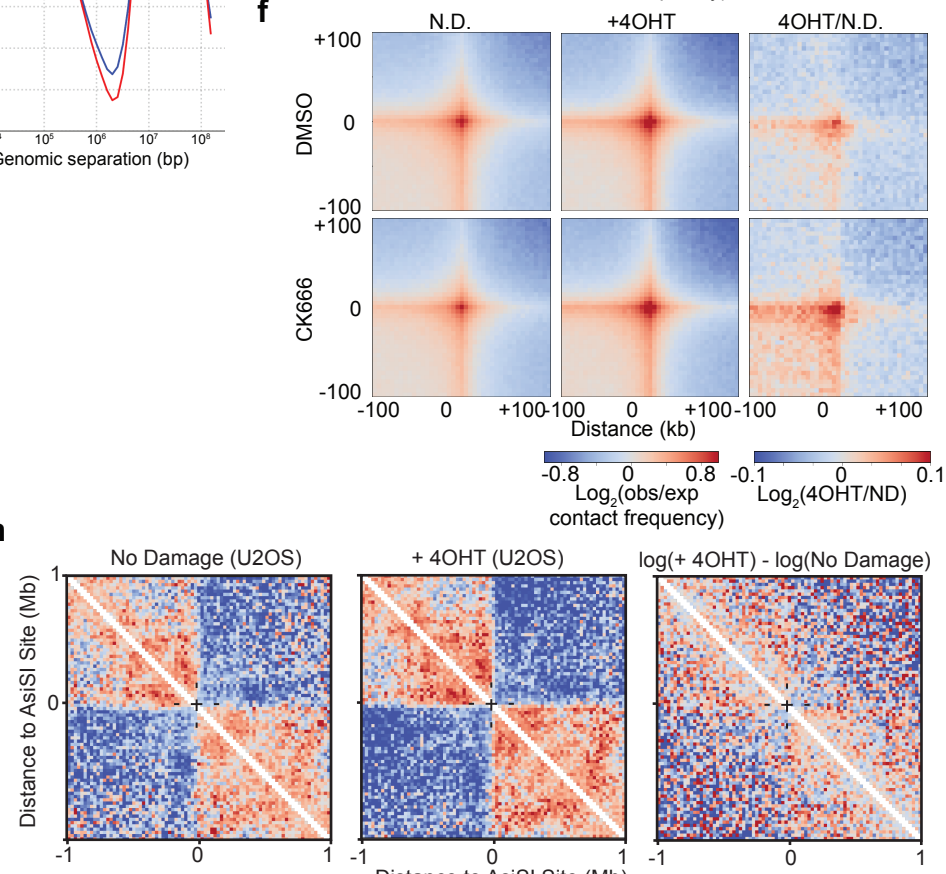

$\log (+4 \mathrm{OHT})-\log ($ No Damage $)$

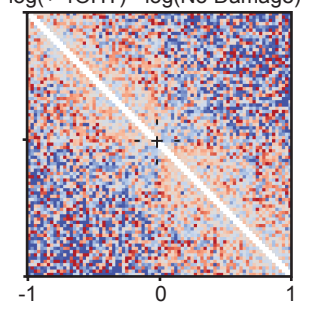

Extended Data Figure 2| Genome reorganization following damage. a, Fraction of the genome (250 kb bins) classified as A (EV1>0) or B (EV1<0) compartment before and after damage +/- CK-666 (100 $\mu M)$. b, Genome wide changes in EV1 (+4OHT vs N.D. control) plotted as a function of binned eigenvalues in the undamaged control. c, Saddle plots representing chromatin compartmentalization, i.e. the strength of AA (bottom right quadrant) and B-B (top left quadrant) compartment interactions versus interactions between compartments ( $250 \mathrm{~kb}$-binned data). Data is normalized by the expected interaction frequency 
based on genomic distance. Histograms along the X-axis show the distribution of saddle strength, as measured by $(A A+B B) /(A B+B A)$. $d$, Top: Contact probability $P$ plotted as a function of genomic distance $s$ $(P(s))$ for chromosome 2 in the presence or absence of DNA damage (4OHT). Bottom: Derivative plots emphasize changes in distant gene-gene interactions. e, Average $\log _{2}$ (observed/expected) $\mathrm{Hi}-\mathrm{C}$ interaction frequency maps in the $200 \mathrm{~kb}$ regions flanking top CTCF sites (4052) binned at $5 \mathrm{~kb}$ resolution. f, $\log _{2}$ (observed/expected) $\mathrm{Hi}-\mathrm{C}$ maps centered on all possible pairwise combinations of the top CTCF sites (11532 interactions) binned at $5 \mathrm{~kb}$ and averaged for a $200 \mathrm{~kb}$ flanking window. Log ratio interaction maps for $4 \mathrm{OHT}$ vs control treatments are shown. $\mathrm{g}$, Gene set enrichment analysis (GSEA) plot (score curves) assessing the enrichment of the frequently cut AsiSI sites in transcriptionally active regions $\mathbf{h}$, Pile-up heat maps $1 \mathrm{Mb}$ surrounding the most frequently cut AsiSI sites in U2OS cells. For these experiments, no damage samples were exponentially growing, and damaged cells were synchronized in G2. Cells synchronized in G1 looked similar (data not shown). 


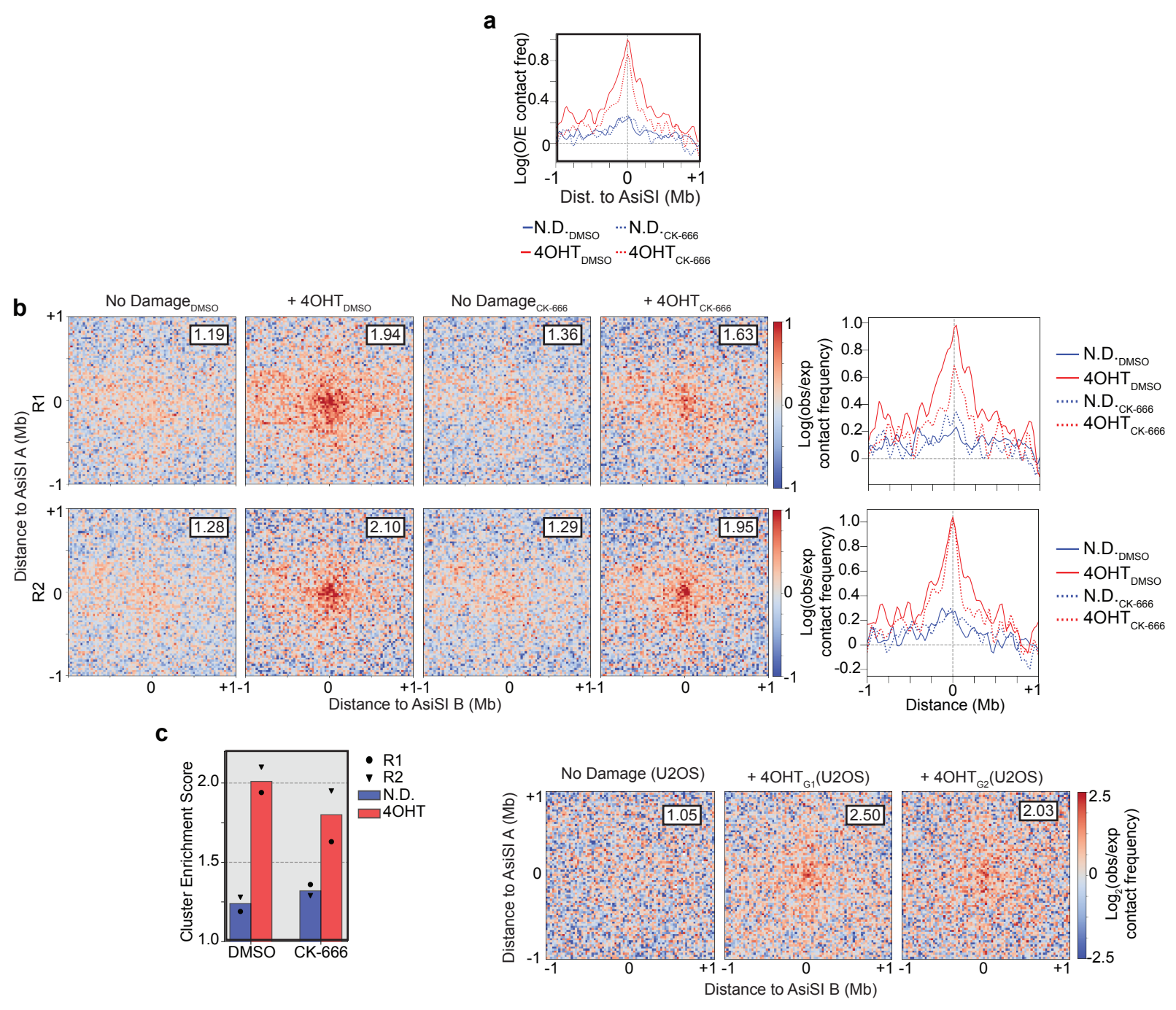

Extended Data Figure 3| DSB clustering in mouse and human cell lines. a, Observed-expected contact frequencies derived from aggregate peak analysis (APA) in the presence and absence of damage $+/-100$ $\mu \mathrm{M}$ CK-666 (MEF cells). b, Left: Average Hi-C interactions centered on all possible combinations of the Top97 AsiSI digested sites in cis (304 interactions between damaged bins) for individual biological replicates. Data is binned at $25 \mathrm{~kb}$ and averaged for a $2 \mathrm{Mb}$ flanking window. $\log _{2}$ (observed/expected) $\mathrm{Hi}$ $\mathrm{C}$ maps are shown in the presence or absence of damage (4OHT) +/- CK-666. Top right corner in each aggregate peak analysis (APA) plot displays cluster enrichment score which is calculated using the ratios of the average interaction frequency of the 9 central bins (125 kb) / average interaction frequency of the outside bins (125 kb-1 Mb). Right: Observed-expected contact frequency derived from aggregate peak analysis (APA) in the presence and absence of damage +/- $100 \mu \mathrm{M}$ CK-666 (MEF cells) for individual biological replicates. c, Quantification of cluster enrichment score for two biological replicates and pooled 
bioRxiv preprint doi: https://doi.org/10.1101/2021.10.22.465487; this version posted October 24, 2021. The copyright holder for this preprint (which was not certified by peer review) is the author/funder, who has granted bioRxiv a license to display the preprint in perpetuity. It is made available under aCC-BY-NC-ND 4.0 International license.

data. d, APA plots ( $2 \mathrm{Mb}$ flanking window, $25 \mathrm{~kb}$ resolution) of interactions between the most frequently cut U2OS AsiSI sites in cis (155 pairs). No damage cells were exponential growing, and damaged (+4OHT) cells were synchronized in G2. Cells synchronized in G1 looked similar (data not shown). 
a

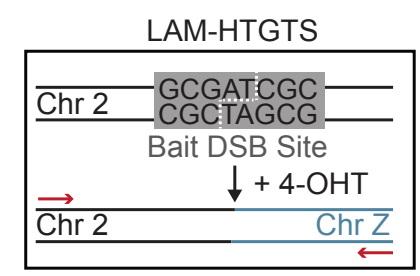

C

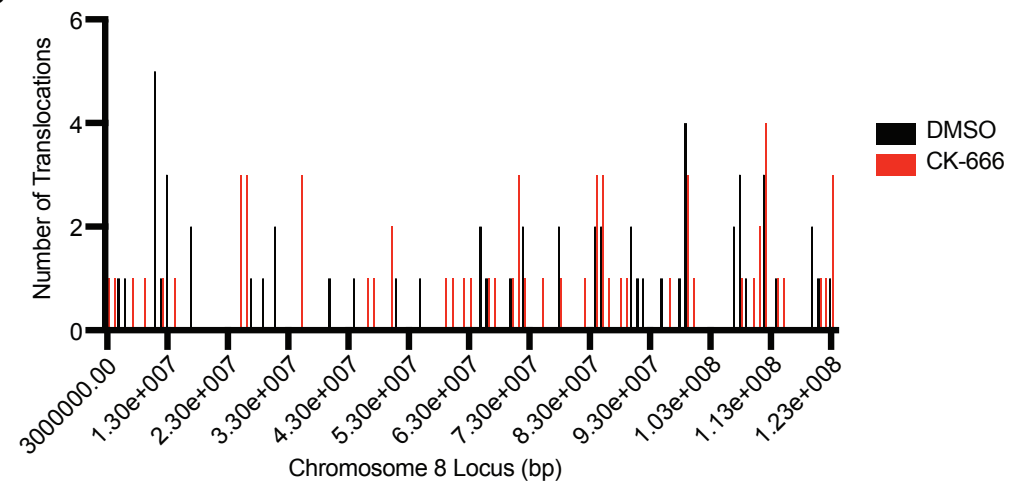

b

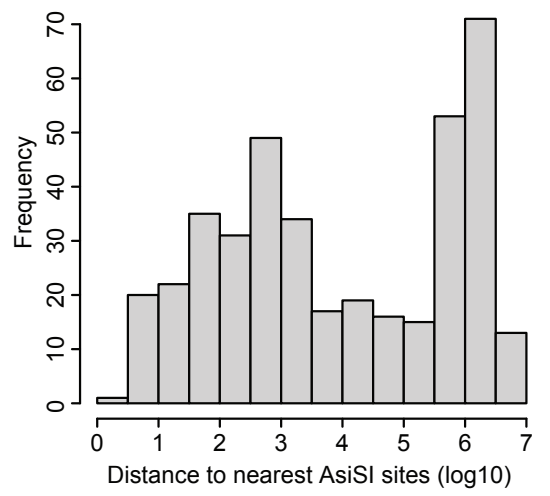

d



e



f

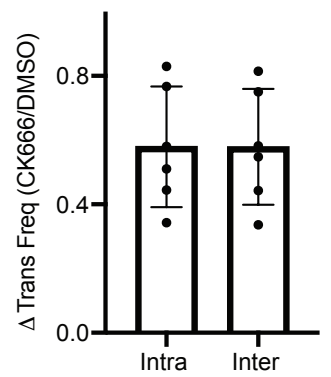

Extended Data Figure 4|Recurrent (proximal) versus spontaneous (distal) translocations a, Schematic of HTGTS experiment. The bait site is located on chromosome 2, 13271321 bp. b, Distance of translocating loci (prey) to the nearest AsiSI motif in U2OS cells. $\mathbf{b}$, Distribution of distal prey along chromosome 8 in two individual libraries (+/- CK-666). c, Distribution of proximal and distal prey into promoter, gene body, and intergenic categories. d, Gene set enrichment analysis (GSEA) plot (score curves) assessing enrichment of translocating prey in transcriptionally active areas. e, Fold change in normalized translocation frequency for intra- and inter-chromosomal events. Mean and standard deviation. 
a

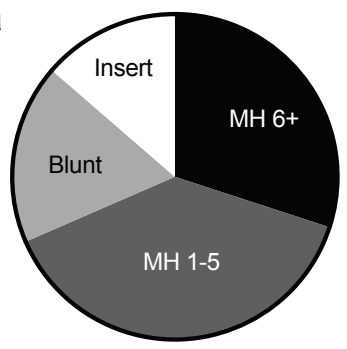

b

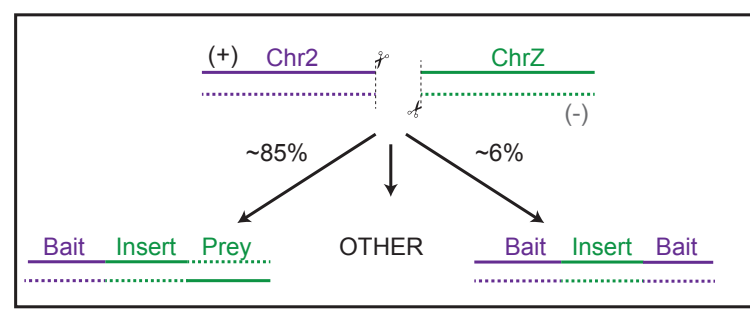

C

\begin{tabular}{|c|c|}
\hline chr2: $13271278-13271321$ & chr11: 120784104-120784104 \\
\hline \multicolumn{2}{|c|}{ GCGAAAACAGGATCCCGCAGCAGGAACACACCCTGTCCCAGCGATAGCATGAGCCGTGCGCGGCGGGTGTTGTGCCAGGACCGCGGCCAGCGCGAGGCAGGCCCGG... } \\
\hline chr2: $13271278-13271325$ & chr7: $44246861-44246914$ \\
\hline \multicolumn{2}{|c|}{ CGATGTCGAAAACAGGATCCCGCAGCAGGAACACACCCTGTCCCAGCGATCGCGTGTGAGGTGAGCTCTGTCCCGGTGAGTTTTGCCACGGACACCTCGGGACTCGTGTTTCC } \\
\hline chr2: $13271278-13271313$ & chr2: $64098493-64098533$ \\
\hline
\end{tabular}

Extended Data Figure 5 |Junctional analysis of translocation events. a, Distribution of HTGTS prey by junctional type ( $\mathrm{MH}$, microhomology; blunt end ligation; insertion). b, Schematic representation of insertion events. c, Example reads that contain an insertion event (underline) in between bait (purple) and prey (green). 


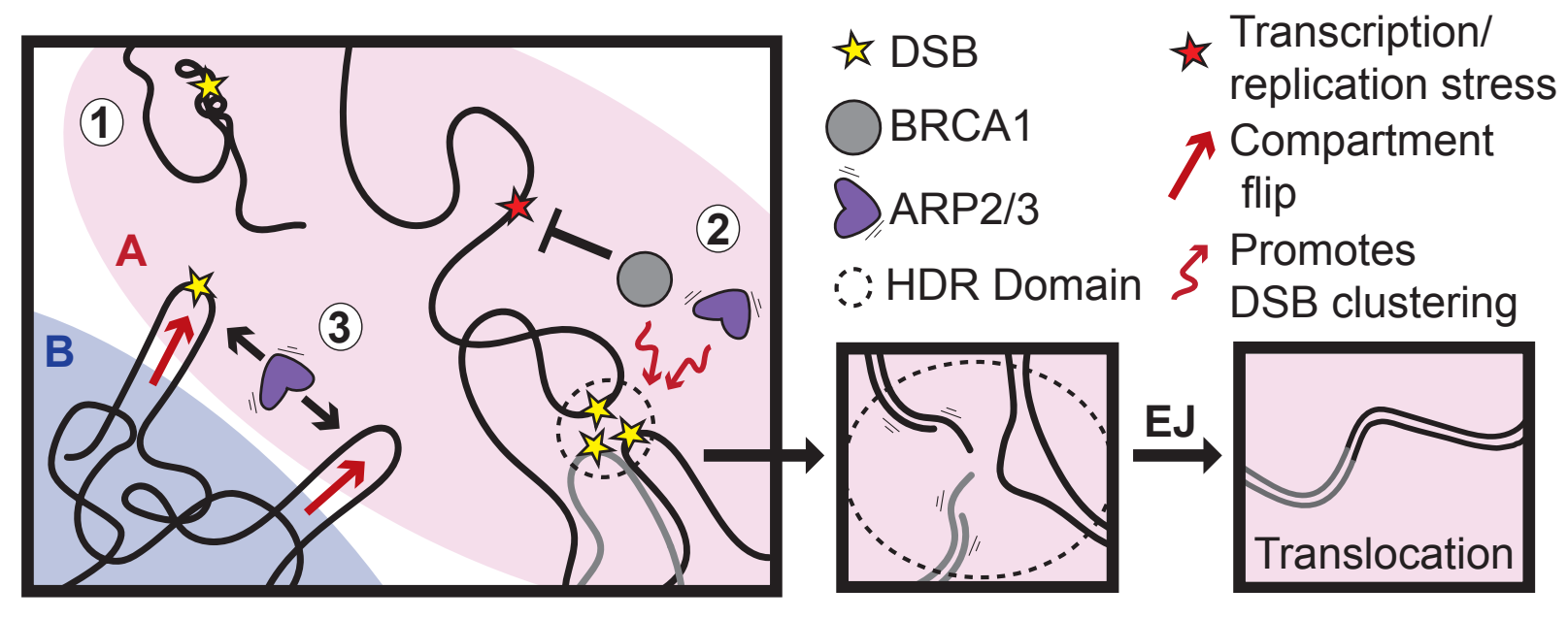

Extended Data Figure 7|Genome reorganization following DNA damage facilitates translocations.

Schematic representation of the multiscale changes in the 3D genome following damage. 


\section{Works Cited}

1 Schrank, B. \& Gautier, J. Assembling nuclear domains: Lessons from DNA repair. J Cell Biol 218, 2444-2455, doi:10.1083/jcb.201904202 (2019).

2 Schrank, B. R. et al. Nuclear ARP2/3 drives DNA break clustering for homology-directed repair. Nature 559, 61-66, doi:10.1038/s41586-018-0237-5 (2018).

3 Symington, L. S. \& Gautier, J. Double-strand break end resection and repair pathway choice. Annu Rev Genet 45, 247-271, doi:10.1146/annurev-genet-110410-132435 (2011).

4 Caridi, C. P. et al. Nuclear F-actin and myosins drive relocalization of heterochromatic breaks. Nature 559, 54-60, doi:10.1038/s41586-018-0242-8 (2018).

5 Lisby, M., Mortensen, U. H. \& Rothstein, R. Colocalization of multiple DNA double-strand breaks at a single Rad52 repair centre. Nat Cell Biol 5, 572-577, doi:10.1038/ncb997 (2003).

6 Aten, J. A. et al. Dynamics of DNA double-strand breaks revealed by clustering of damaged chromosome domains. Science 303, 92-95, doi:10.1126/science.1088845 (2004).

$7 \quad$ Neumaier, T. et al. Evidence for formation of DNA repair centers and dose-response nonlinearity in human cells. Proc Natl Acad Sci U S A 109, 443-448, doi:10.1073/pnas.1117849108 (2012).

8 Caron, P. et al. Non-redundant Functions of ATM and DNA-PKcs in Response to DNA DoubleStrand Breaks. Cell Rep 13, 1598-1609, doi:10.1016/j.celrep.2015.10.024 (2015).

9 Roukos, V. et al. Spatial dynamics of chromosome translocations in living cells. Science 341, 660664, doi:10.1126/science.1237150 (2013).

10 Sunder, S. \& Wilson, T. E. Frequency of DNA end joining in trans is not determined by the predamage spatial proximity of double-strand breaks in yeast. Proc Natl Acad Sci U S A, doi:10.1073/pnas.1818595116 (2019).

11 Zhang, Y. \& Jasin, M. An essential role for CtIP in chromosomal translocation formation through an alternative end-joining pathway. Nat Struct Mol Biol 18, 80-84, doi:10.1038/nsmb.1940 (2011).

12 Rogakou, E. P., Boon, C., Redon, C. \& Bonner, W. M. Megabase chromatin domains involved in DNA double-strand breaks in vivo. J Cell Biol 146, 905-916, doi:10.1083/jcb.146.5.905 (1999).

13 lacovoni, J. S. et al. High-resolution profiling of gammaH2AX around DNA double strand breaks in the mammalian genome. EMBO J 29, 1446-1457, doi:10.1038/emboj.2010.38 (2010).

14 Clouaire, T. et al. Comprehensive Mapping of Histone Modifications at DNA Double-Strand Breaks Deciphers Repair Pathway Chromatin Signatures. Mol Cell 72, 250-262 e256, doi:10.1016/j.molcel.2018.08.020 (2018).

15 Aymard, F. et al. Genome-wide mapping of long-range contacts unveils clustering of DNA doublestrand breaks at damaged active genes. Nat Struct Mol Biol 24, 353-361, doi:10.1038/nsmb.3387 (2017).

16 Callen, E. et al. 53BP1 Enforces Distinct Pre- and Post-resection Blocks on Homologous Recombination. Mol Cell 77, 26-38 e27, doi:10.1016/j.molcel.2019.09.024 (2020).

17 Aymard, F. et al. Transcriptionally active chromatin recruits homologous recombination at DNA double-strand breaks. Nat Struct Mol Biol 21, 366-374, doi:10.1038/nsmb.2796 (2014).

18 Lieberman-Aiden, E. et al. Comprehensive mapping of long-range interactions reveals folding principles of the human genome. Science 326, 289-293, doi:10.1126/science.1181369 (2009).

19 Imakaev, M. et al. Iterative correction of $\mathrm{Hi}-\mathrm{C}$ data reveals hallmarks of chromosome organization. Nat Methods 9, 999-1003, doi:10.1038/nmeth.2148 (2012).

20 Oudelaar, A. M. \& Higgs, D. R. The relationship between genome structure and function. Nat Rev Genet 22, 154-168, doi:10.1038/s41576-020-00303-x (2021).

21 Nora, E. P. et al. Targeted Degradation of CTCF Decouples Local Insulation of Chromosome Domains from Genomic Compartmentalization. Cell 169, 930-944 e922, doi:10.1016/j.cell.2017.05.004 (2017). 
Gassler, J. et al. A mechanism of cohesin-dependent loop extrusion organizes zygotic genome architecture. EMBO J 36, 3600-3618, doi:10.15252/embj.201798083 (2017).

Kim, J. S., Krasieva, T. B., LaMorte, V., Taylor, A. M. \& Yokomori, K. Specific recruitment of human cohesin to laser-induced DNA damage. J Biol Chem 277, 45149-45153, doi:10.1074/jbc.M209123200 (2002).

24 Strom, L., Lindroos, H. B., Shirahige, K. \& Sjogren, C. Postreplicative recruitment of cohesin to double-strand breaks is required for DNA repair. Mol Cell 16, 1003-1015, doi:10.1016/j.molcel.2004.11.026 (2004).

25 Arnould, C. et al. Loop extrusion as a mechanism for formation of DNA damage repair foci. Nature 590, 660-665, doi:10.1038/s41586-021-03193-z (2021).

26 Sanders, J. T. et al. Radiation-induced DNA damage and repair effects on 3D genome organization. Nat Commun 11, 6178, doi:10.1038/s41467-020-20047-w (2020).

27 Lajoie, B. R., Dekker, J. \& Kaplan, N. The Hitchhiker's guide to Hi-C analysis: practical guidelines. Methods 72, 65-75, doi:10.1016/j.ymeth.2014.10.031 (2015).

28 Rao, S. S. et al. A 3D map of the human genome at kilobase resolution reveals principles of chromatin looping. Cell 159, 1665-1680, doi:10.1016/j.cell.2014.11.021 (2014).

Klein, I. A. et al. Translocation-capture sequencing reveals the extent and nature of chromosomal rearrangements in B lymphocytes. Cell 147, 95-106, doi:10.1016/j.cell.2011.07.048 (2011).

Bunting, S. F. \& Nussenzweig, A. End-joining, translocations and cancer. Nat Rev Cancer 13, 443454, doi:10.1038/nrc3537 (2013).

31 Schwer, B. et al. Transcription-associated processes cause DNA double-strand breaks and translocations in neural stem/progenitor cells. Proc Natl Acad Sci U S A 113, 2258-2263, doi:10.1073/pnas.1525564113 (2016).

$32 \mathrm{Hu}$, J. et al. Detecting DNA double-stranded breaks in mammalian genomes by linear amplification-mediated high-throughput genome-wide translocation sequencing. Nat Protoc 11, 853-871, doi:10.1038/nprot.2016.043 (2016).

33 Hamperl, S. \& Cimprich, K. A. Conflict Resolution in the Genome: How Transcription and Replication Make It Work. Cell 167, 1455-1467, doi:10.1016/j.cell.2016.09.053 (2016).

34 Chiarle, R. et al. Genome-wide translocation sequencing reveals mechanisms of chromosome breaks and rearrangements in B cells. Cell 147, 107-119, doi:10.1016/j.cell.2011.07.049 (2011). Crowe, J. L. et al. Kinase-dependent structural role of DNA-PKcs during immunoglobulin class switch recombination. Proc Natl Acad Sci U S A 115, 8615-8620, doi:10.1073/pnas.1808490115 (2018).

36 Seol, J. H., Shim, E. Y. \& Lee, S. E. Microhomology-mediated end joining: Good, bad and ugly. Mutat Res 809, 81-87, doi:10.1016/j.mrfmmm.2017.07.002 (2018).

37 Smith, M. J., Bryant, E. E., Joseph, F. J. \& Rothstein, R. DNA damage triggers increased mobility of chromosomes in G1-phase cells. Mol Biol Cell 30, 2620-2625, doi:10.1091/mbc.E19-08-0469 (2019).

38 Lal, A. et al. Comprehensive genomic characterization of breast tumors with BRCA1 and BRCA2 mutations. BMC Med Genomics 12, 84, doi:10.1186/s12920-019-0545-0 (2019).

39 Davies, H. et al. HRDetect is a predictor of BRCA1 and BRCA2 deficiency based on mutational signatures. Nat Med 23, 517-525, doi:10.1038/nm.4292 (2017).

40 Zamborszky, J. et al. Loss of BRCA1 or BRCA2 markedly increases the rate of base substitution mutagenesis and has distinct effects on genomic deletions. Oncogene 36, 5085-5086, doi:10.1038/onc.2017.213 (2017).

41 Bunting, S. F. et al. 53BP1 inhibits homologous recombination in Brca1-deficient cells by blocking resection of DNA breaks. Cell 141, 243-254, doi:10.1016/j.cell.2010.03.012 (2010). 
Tarsounas, M. \& Sung, P. The antitumorigenic roles of BRCA1-BARD1 in DNA repair and replication. Nat Rev Mol Cell Biol 21, 284-299, doi:10.1038/s41580-020-0218-z (2020).

43 Chen, C. C., Feng, W., Lim, P. X., Kass, E. M. \& Jasin, M. Homology-Directed Repair and the Role of BRCA1, BRCA2, and Related Proteins in Genome Integrity and Cancer. Annu Rev Cancer Biol 2, 313-336, doi:10.1146/annurev-cancerbio-030617-050502 (2018).

44 Sirbu, B. M. \& Cortez, D. DNA damage response: three levels of DNA repair regulation. Cold Spring Harb Perspect Biol 5, a012724, doi:10.1101/cshperspect.a012724 (2013).

45 Van, H. T. \& Santos, M. A. Histone modifications and the DNA double-strand break response. Cell Cycle 17, 2399-2410, doi:10.1080/15384101.2018.1542899 (2018).

46 Benedict, B. et al. WAPL-Dependent Repair of Damaged DNA Replication Forks Underlies Oncogene-Induced Loss of Sister Chromatid Cohesion. Dev Cell 52, 683-698 e687, doi:10.1016/j.devcel.2020.01.024 (2020).

47 Zhang, X. et al. Fundamental roles of chromatin loop extrusion in antibody class switching. Nature 575, 385-389, doi:10.1038/s41586-019-1723-0 (2019).

48 Dai, H. Q. et al. Loop extrusion mediates physiological Igh locus contraction for RAG scanning. Nature 590, 338-343, doi:10.1038/s41586-020-03121-7 (2021).

49 Belin, B. J., Lee, T. \& Mullins, R. D. DNA damage induces nuclear actin filament assembly by Formin -2 and Spire-(1/2) that promotes efficient DNA repair. [corrected]. Elife 4, e07735, doi:10.7554/eLife.07735 (2015).

50 Lottersberger, F., Karssemeijer, R. A., Dimitrova, N. \& de Lange, T. 53BP1 and the LINC Complex Promote Microtubule-Dependent DSB Mobility and DNA Repair. Cell 163, 880-893, doi:10.1016/j.cell.2015.09.057 (2015).

51 Altmeyer, M. et al. Liquid demixing of intrinsically disordered proteins is seeded by poly(ADPribose). Nat Commun 6, 8088, doi:10.1038/ncomms9088 (2015).

52 Kilic, S. et al. Phase separation of 53BP1 determines liquid-like behavior of DNA repair compartments. EMBO J 38, e101379, doi:10.15252/embj.2018101379 (2019).

53 Gostissa, M., Alt, F. W. \& Chiarle, R. Mechanisms that promote and suppress chromosomal translocations in lymphocytes. Annu Rev Immunol 29, 319-350, doi:10.1146/annurev-immunol031210-101329 (2011).

54 Piazza, A., Wright, W. D. \& Heyer, W. D. Multi-invasions Are Recombination Byproducts that Induce Chromosomal Rearrangements. Cell 170, 760-773 e715, doi:10.1016/j.cell.2017.06.052 (2017).

55 Weinstock, D. M., Brunet, E. \& Jasin, M. Induction of chromosomal translocations in mouse and human cells using site-specific endonucleases. J Natl Cancer Inst Monogr, 20-24, doi:10.1093/jncimonographs/Ign009 (2008).

56 Goloborodko, A., Abdennur, N., Venev, S., hbbrandao \& gfudenberg. mirnylab/pairtools v0.3.0. doi:doi:10.5281/zenodo.2649383. (2019).

57 Abdennur, N. \& Mirny, L. A. Cooler: scalable storage for Hi-C data and other genomically labeled arrays. Bioinformatics 36, 311-316, doi:10.1093/bioinformatics/btz540 (2020).

58 Venev, S. et al. mirnylab/cooltools: v0.3.2. doi:10.5281/zenodo.3787004 (2020).

59 Venev, S. et al. open2c/cooltools: v0.4.1. doi:10.5281/zenodo.5214125 (2021).

60 Canela, A. et al. Genome Organization Drives Chromosome Fragility. Cell 170, 507-521 e518, doi:10.1016/j.cell.2017.06.034 (2017).

61 Heinz, S. et al. Simple combinations of lineage-determining transcription factors prime cisregulatory elements required for macrophage and B cell identities. Mol Cell 38, 576-589, doi:10.1016/j.molcel.2010.05.004 (2010).

$62 \mathrm{Kim}, \mathrm{T}$. H. et al. Analysis of the vertebrate insulator protein CTCF-binding sites in the human genome. Cell 128, 1231-1245, doi:10.1016/j.cell.2006.12.048 (2007). 
bioRxiv preprint doi: https://doi.org/10.1101/2021.10.22.465487; this version posted October 24, 2021. The copyright holder for this preprint

(which was not certified by peer review) is the author/funder, who has granted bioRxiv a license to display the preprint in perpetuity. It is made available under aCC-BY-NC-ND 4.0 International license.

63 Yu, G., Wang, L. G. \& He, Q. Y. ChIPseeker: an R/Bioconductor package for ChIP peak annotation, comparison and visualization. Bioinformatics 31, 2382-2383, doi:10.1093/bioinformatics/btv145 (2015).

64 Wong, N., John, S., Nussenzweig, A. \& Canela, A. END-seq: An Unbiased, High-Resolution, and Genome-Wide Approach to Map DNA Double-Strand Breaks and Resection in Human Cells. Methods Mol Biol 2153, 9-31, doi:10.1007/978-1-0716-0644-5_2 (2021).

65 Shen, Y. et al. A map of the cis-regulatory sequences in the mouse genome. Nature 488, 116-120, doi:10.1038/nature11243 (2012). 